\title{
Study of SHMT2 inhibitors and their Binding Mechanism by Computational
}

\author{
Alanine Scanning \\ Liping $\mathrm{He}^{1}$, Jingxiao Bao ${ }^{1}$, Yunpeng Yang ${ }^{1}$, Suzhen Dong ${ }^{1}$, Lujia Zhang ${ }^{1,2}$, Yifei $\mathrm{Qi}^{1,2 *}$, and John Z.H. \\ Zhang ${ }^{1,2,3^{*}}$ \\ ${ }^{1}$ Shanghai Engineering Research Center of Molecular Therapeutics \& New Drug Development, Shanghai Key \\ Laboratory of Green Chemistry \& Chemical Process, School of Chemistry and Molecular Engineering, East \\ China Normal University, Shanghai 200062, China \\ ${ }^{2} N Y U-E C N U$ Center for Computational Chemistry at NYU Shanghai, Shanghai 200062, China \\ ${ }^{3}$ Department of Chemistry, New York University, NY, NY 10003, USA
}

${ }^{*}$ Corresponding Authors

E-mail: yfqi@,chem.ecnu.edu.cn, John.zhang@nyu.edu 


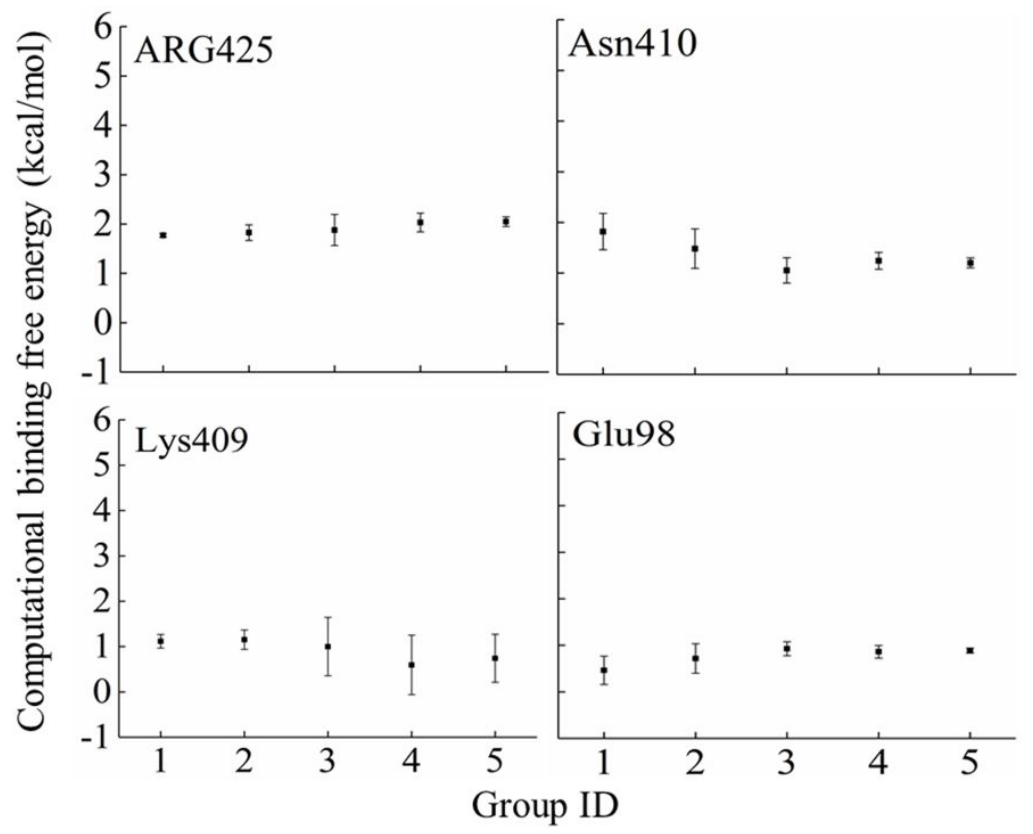

Figure S1. Average residue specific contributions to the total binding free energy in the five groups of inhibitors with different magnitude of binding affinities. 
Table S1. Structures of the twenty-eight compounds and their experimental binding energies.

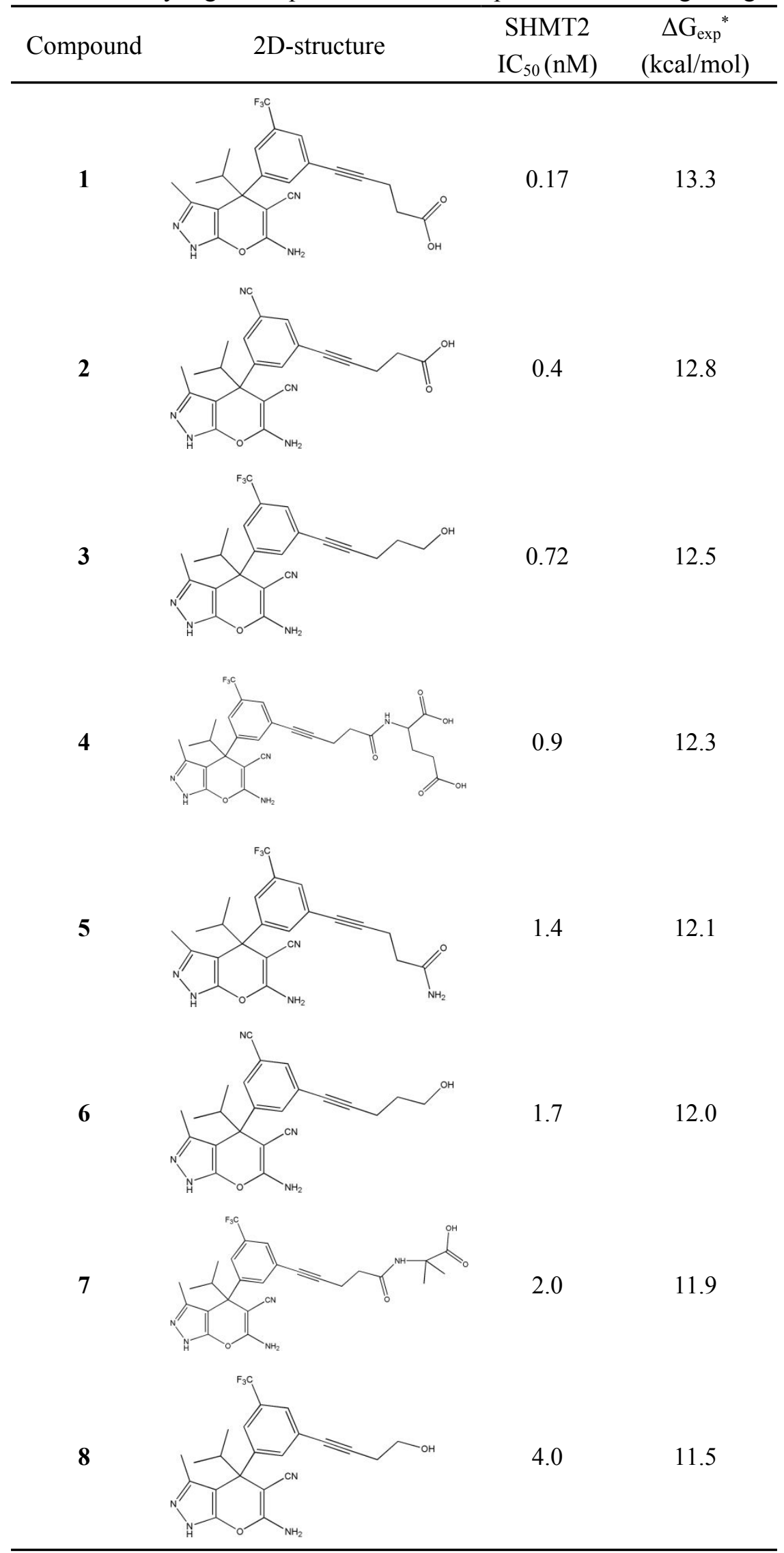




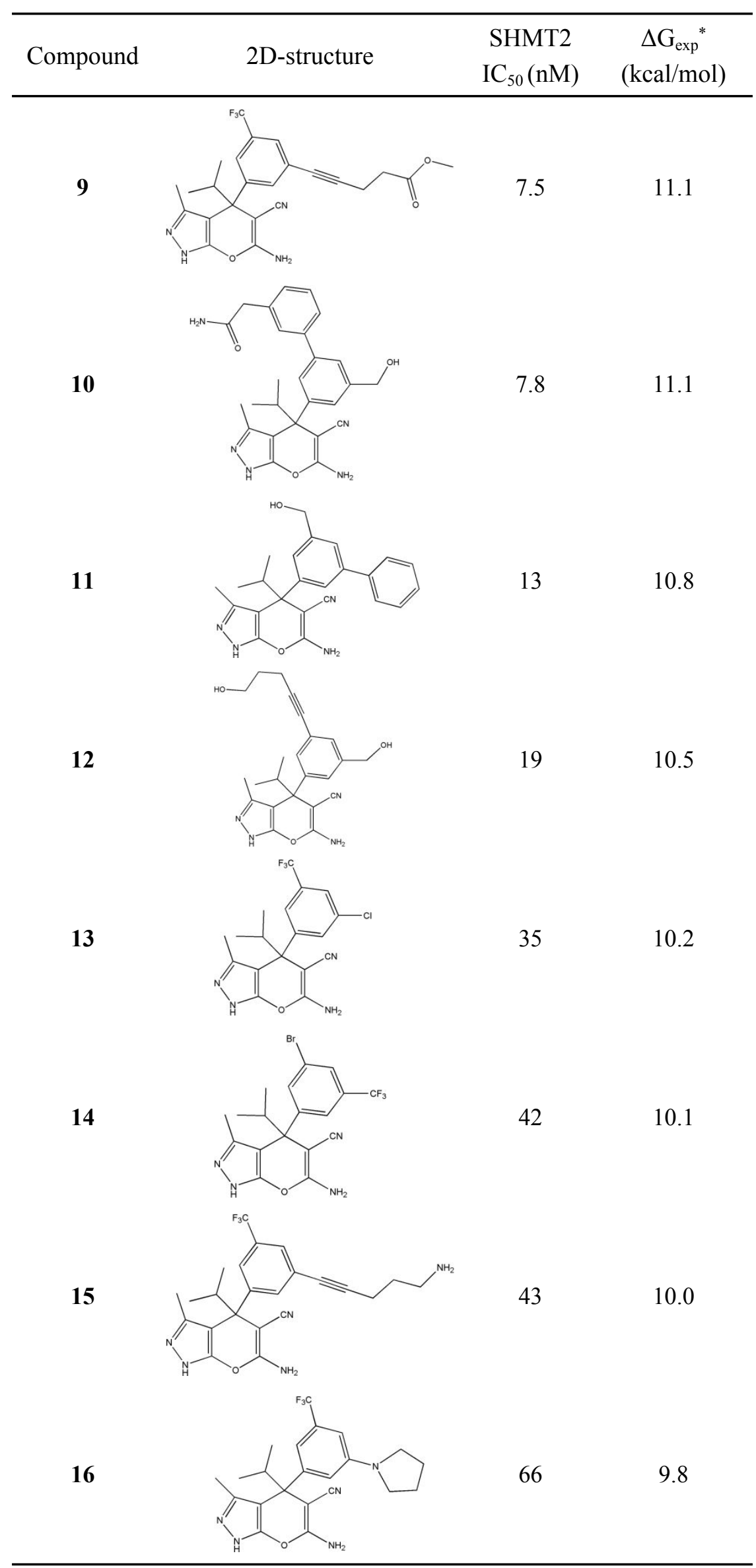




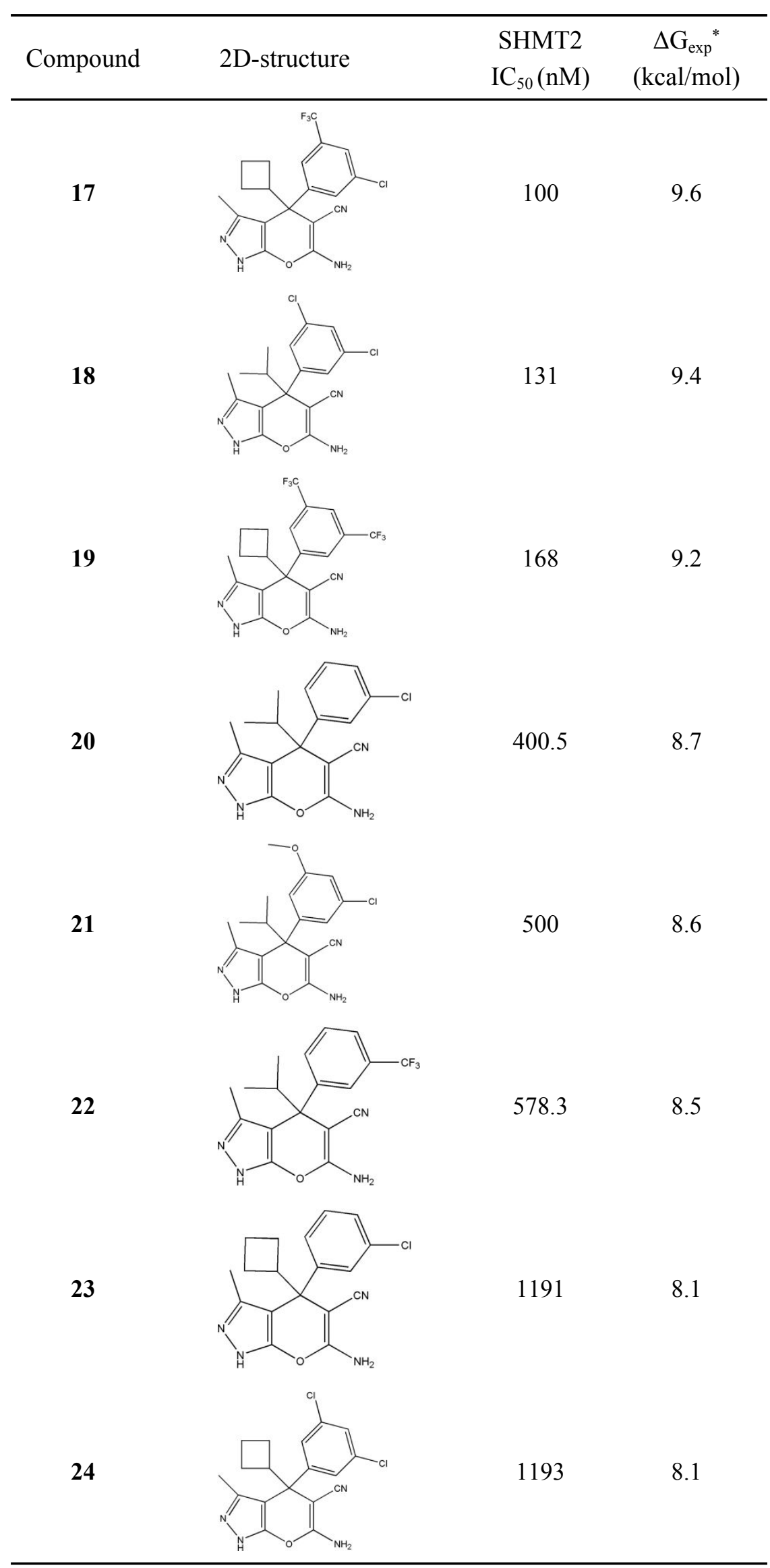




Compound


Table S2. Residue-specific binding free energies of SHMT2 and compound 1 from AS-IE. Only residues with $\Delta \Delta G_{\text {bind }}^{x \rightarrow a}>0.2 \mathrm{kcal} / \mathrm{mol}$. are listed and all values are in $\mathrm{kcal} / \mathrm{mol}$.

\begin{tabular}{|c|c|c|c|c|c|c|c|}
\hline Residue & $\Delta \Delta \mathrm{E}_{\mathrm{vdw}}$ & $\Delta \Delta \mathrm{E}_{\text {ele }}$ & $\Delta \Delta \mathrm{GB}$ & $\Delta \Delta \mathrm{NP}$ & $\Delta \Delta \mathrm{H}$ & IE & $\Delta \Delta G_{\text {bind }}^{x \rightarrow a}$ \\
\hline Y105'A & $5.6 \pm 0.2$ & $0.1 \pm 0.2$ & $-0.7 \pm 0.1$ & $0.5 \pm 0.0$ & $5.5 \pm 0.1$ & $-0.5 \pm 0.1$ & $4.9 \pm 0.1$ \\
\hline L166A & $3.4 \pm 0.1$ & $0.1 \pm 0.0$ & $-0.2 \pm 0.1$ & $0.2 \pm 0.0$ & $3.4 \pm 0.1$ & $-0.5 \pm 0.3$ & $2.9 \pm 0.4$ \\
\hline R425A & $2.0 \pm 0.3$ & $-0.3 \pm 0.1$ & $0.2 \pm 0.1$ & $0.1 \pm 0.0$ & $2.0 \pm 0.2$ & $-0.1 \pm 0.1$ & $1.9 \pm 0.2$ \\
\hline L172A & $2.0 \pm 0.2$ & $0.0 \pm 0.0$ & $-0.1 \pm 0.0$ & $0.1 \pm 0.0$ & $2.0 \pm 0.2$ & $-0.2 \pm 0.1$ & $1.8 \pm 0.2$ \\
\hline H171A & $1.2 \pm 0.1$ & $-0.1 \pm 0.1$ & $0.1 \pm 0.0$ & $0.0 \pm 0.0$ & $1.2 \pm 0.1$ & $0.1 \pm 0.0$ & $1.3 \pm 0.1$ \\
\hline Y176A & $1.5 \pm 0.1$ & $-0.1 \pm 0.0$ & $-0.1 \pm 0.0$ & $0.1 \pm 0.0$ & $1.4 \pm 0.1$ & $-0.2 \pm 0.1$ & $1.3 \pm 0.2$ \\
\hline K409A & $1.1 \pm 0.3$ & $2.3 \pm 0.0$ & $-2.3 \pm 0.0$ & $0.3 \pm 0.0$ & $1.4 \pm 0.3$ & $-0.2 \pm 0.1$ & $1.2 \pm 0.4$ \\
\hline N410A & $1.3 \pm 0.2$ & $0.6 \pm 0.2$ & $-0.6 \pm 0.1$ & $0.1 \pm 0.0$ & $1.4 \pm 0.3$ & $-0.3 \pm 0.2$ & $1.1 \pm 0.1$ \\
\hline E98'A & $1.3 \pm 0.2$ & $0.3 \pm 0.1$ & $-0.7 \pm 0.1$ & $0.2 \pm 0.0$ & $1.0 \pm 0.1$ & $-0.1 \pm 0.0$ & $1.0 \pm 0.2$ \\
\hline N408A & $1.1 \pm 0.1$ & $0.1 \pm 0.1$ & $-0.4 \pm 0.0$ & $0.1 \pm 0.0$ & $0.9 \pm 0.2$ & $-0.1 \pm 0.1$ & $0.8 \pm 0.2$ \\
\hline I183A & $1.1 \pm 0.1$ & $-0.1 \pm 0.0$ & $-0.3 \pm 0.1$ & $0.1 \pm 0.0$ & $0.9 \pm 0.0$ & $-0.3 \pm 0.1$ & $0.6 \pm 0.1$ \\
\hline S226A & $0.5 \pm 0.0$ & $0.3 \pm 0.1$ & $-0.1 \pm 0.0$ & $0.0 \pm 0.0$ & $0.7 \pm 0.1$ & $-0.1 \pm 0.1$ & $0.6 \pm 0.2$ \\
\hline F320'A & $0.7 \pm 0.1$ & $0.0 \pm 0.0$ & $-0.2 \pm 0.0$ & $0.0 \pm 0.0$ & $0.5 \pm 0.1$ & $0.0 \pm 0.0$ & $0.5 \pm 0.1$ \\
\hline F317'A & $0.5 \pm 0.1$ & $0.1 \pm 0.0$ & $-0.3 \pm 0.1$ & $0.0 \pm 0.0$ & $0.4 \pm 0.1$ & $-0.1 \pm 0.0$ & $0.3 \pm 0.1$ \\
\hline I74A & $0.1 \pm 0.0$ & $0.0 \pm 0.0$ & $0.1 \pm 0.0$ & $0.0 \pm 0.0$ & $0.2 \pm 0.0$ & $0.0 \pm 0.0$ & $0.2 \pm 0.0$ \\
\hline Total & $23.4 \pm 0.4$ & $3.2 \pm 0.1$ & $-5.6 \pm 0.2$ & $1.9 \pm 0.0$ & $22.8 \pm 0.5$ & $-2.5 \pm 0.2$ & $20.4 \pm 0.6$ \\
\hline
\end{tabular}


Table S3. Same as Table S1 but for compound 2.

\begin{tabular}{cccccccc}
\hline Residue & $\Delta \Delta \mathrm{E}_{\mathrm{vdw}}$ & $\Delta \Delta \mathrm{E}_{\text {ele }}$ & $\Delta \Delta \mathrm{GB}$ & $\Delta \Delta \mathrm{NP}$ & $\Delta \Delta \mathrm{H}$ & $\mathrm{IE}$ & $\Delta \Delta G_{\text {bind }}^{x \rightarrow a}$ \\
\hline Y105'A & $5.4 \pm 0.2$ & $0.2 \pm 0.0$ & $-0.7 \pm 0.0$ & $0.5 \pm 0.0$ & $5.3 \pm 0.2$ & $-0.5 \pm 0.1$ & $4.8 \pm 0.3$ \\
L166A & $3.1 \pm 0.1$ & $0.1 \pm 0.0$ & $-0.3 \pm 0.0$ & $0.2 \pm 0.0$ & $3.1 \pm 0.1$ & $-0.7 \pm 0.2$ & $2.4 \pm 0.3$ \\
$\mathrm{R} 425 \mathrm{~A}$ & $2.2 \pm 0.1$ & $-0.5 \pm 0.1$ & $0.4 \pm 0.1$ & $0.1 \pm 0.0$ & $2.2 \pm 0.1$ & $-0.1 \pm 0.0$ & $2.0 \pm 0.1$ \\
$\mathrm{~L} 172 \mathrm{~A}$ & $1.9 \pm 0.1$ & $0.0 \pm 0.0$ & $-0.1 \pm 0.0$ & $0.1 \pm 0.0$ & $1.9 \pm 0.1$ & $-0.2 \pm 0.0$ & $1.7 \pm 0.1$ \\
$\mathrm{Y} 176 \mathrm{~A}$ & $1.6 \pm 0.1$ & $-0.2 \pm 0.2$ & $-0.2 \pm 0.0$ & $0.2 \pm 0.0$ & $1.5 \pm 0.1$ & $-0.3 \pm 0.0$ & $1.2 \pm 0.1$ \\
$\mathrm{~N} 410 \mathrm{~A}$ & $1.3 \pm 0.1$ & $1.0 \pm 0.4$ & $-0.8 \pm 0.2$ & $0.1 \pm 0.0$ & $1.5 \pm 0.2$ & $-0.4 \pm 0.1$ & $1.2 \pm 0.1$ \\
$\mathrm{H} 171 \mathrm{~A}$ & $1.0 \pm 0.1$ & $-0.2 \pm 0.0$ & $0.2 \pm 0.1$ & $0.0 \pm 0.0$ & $1.1 \pm 0.1$ & $0.0 \pm 0.1$ & $1.1 \pm 0.1$ \\
$\mathrm{I} 183 \mathrm{~A}$ & $1.3 \pm 0.1$ & $-0.1 \pm 0.0$ & $-0.3 \pm 0.1$ & $0.1 \pm 0.0$ & $1.1 \pm 0.0$ & $-0.1 \pm 0.0$ & $1.0 \pm 0.1$ \\
$\mathrm{E} 98^{\prime} \mathrm{A}$ & $1.1 \pm 0.2$ & $0.4 \pm 0.0$ & $-0.7 \pm 0.1$ & $0.2 \pm 0.0$ & $0.9 \pm 0.1$ & $-0.1 \pm 0.0$ & $0.8 \pm 0.1$ \\
N408A & $0.9 \pm 0.2$ & $0.1 \pm 0.1$ & $-0.4 \pm 0.0$ & $0.0 \pm 0.0$ & $0.7 \pm 0.2$ & $-0.2 \pm 0.1$ & $0.5 \pm 0.3$ \\
F320'A & $0.6 \pm 0.1$ & $0.0 \pm 0.0$ & $-0.2 \pm 0.0$ & $0.0 \pm 0.0$ & $0.5 \pm 0.1$ & $0.0 \pm 0.0$ & $0.5 \pm 0.1$ \\
S226A & $0.5 \pm 0.0$ & $0.2 \pm 0.1$ & $-0.1 \pm 0.0$ & $0.0 \pm 0.0$ & $0.5 \pm 0.1$ & $-0.1 \pm 0.0$ & $0.4 \pm 0.0$ \\
F317'A & $0.4 \pm 0.0$ & $0.0 \pm 0.0$ & $-0.1 \pm 0.1$ & $0.0 \pm 0.0$ & $0.3 \pm 0.0$ & $0.0 \pm 0.0$ & $0.3 \pm 0.0$ \\
T411A & $0.3 \pm 0.0$ & $-0.5 \pm 0.1$ & $0.3 \pm 0.1$ & $0.0 \pm 0.0$ & $0.2 \pm 0.0$ & $0.0 \pm 0.1$ & $0.2 \pm 0.0$ \\
I74A & $0.1 \pm 0.0$ & $0.0 \pm 0.0$ & $0.1 \pm 0.0$ & $0.0 \pm 0.0$ & $0.2 \pm 0.0$ & $0.0 \pm 0.0$ & $0.2 \pm 0.0$ \\
E397A & $0.2 \pm 0.0$ & $-1.4 \pm 0.0$ & $1.4 \pm 0.0$ & $0.0 \pm 0.0$ & $0.2 \pm 0.0$ & $0.0 \pm 0.0$ & $0.2 \pm 0.0$ \\
H254A & $0.2 \pm 0.0$ & $0.0 \pm 0.0$ & $0.0 \pm 0.0$ & $0.0 \pm 0.0$ & $0.2 \pm 0.0$ & $0.0 \pm 0.0$ & $0.2 \pm 0.0$ \\
\hline Total & $22.1 \pm 0.3$ & $-0.9 \pm 0.2$ & $-1.5 \pm 0.2$ & $1.6 \pm 0.1$ & $21.3 \pm 0.2$ & $-2.6 \pm 0.3$ & $18.7 \pm 0.5$ \\
\hline
\end{tabular}


Table S4. Same as Table S1 but for compound 3.

\begin{tabular}{cccccccc}
\hline Residue & $\Delta \Delta \mathrm{E}_{\mathrm{vdw}}$ & $\Delta \Delta \mathrm{E}_{\text {ele }}$ & $\Delta \Delta \mathrm{GB}$ & $\Delta \Delta \mathrm{NP}$ & $\Delta \Delta \mathrm{H}$ & $\mathrm{IE}$ & $\Delta \Delta G_{\text {bind }}^{x \rightarrow a}$ \\
\hline Y105'A & $5.3 \pm 0.2$ & $0.0 \pm 0.1$ & $-0.7 \pm 0.0$ & $0.5 \pm 0.0$ & $5.1 \pm 0.1$ & $-0.4 \pm 0.4$ & $4.7 \pm 0.1$ \\
L166A & $3.2 \pm 0.1$ & $0.0 \pm 0.0$ & $-0.2 \pm 0.0$ & $0.2 \pm 0.0$ & $3.2 \pm 0.2$ & $-0.6 \pm 0.2$ & $2.6 \pm 0.4$ \\
$\mathrm{R} 425 \mathrm{~A}$ & $2.1 \pm 0.3$ & $-0.3 \pm 0.2$ & $0.2 \pm 0.1$ & $0.1 \pm 0.0$ & $2.1 \pm 0.3$ & $-0.1 \pm 0.0$ & $2.1 \pm 0.3$ \\
$\mathrm{~L} 172 \mathrm{~A}$ & $1.9 \pm 0.3$ & $0.0 \pm 0.0$ & $-0.1 \pm 0.0$ & $0.1 \pm 0.0$ & $1.9 \pm 0.3$ & $-0.1 \pm 0.0$ & $1.8 \pm 0.3$ \\
$\mathrm{~N} 410 \mathrm{~A}$ & $1.4 \pm 0.2$ & $0.7 \pm 0.3$ & $-0.7 \pm 0.2$ & $0.1 \pm 0.0$ & $1.5 \pm 0.2$ & $-0.3 \pm 0.1$ & $1.2 \pm 0.1$ \\
$\mathrm{Y} 176 \mathrm{~A}$ & $1.4 \pm 0.2$ & $0.0 \pm 0.1$ & $-0.2 \pm 0.1$ & $0.2 \pm 0.0$ & $1.4 \pm 0.2$ & $-0.2 \pm 0.1$ & $1.2 \pm 0.2$ \\
$\mathrm{H} 171 \mathrm{~A}$ & $1.1 \pm 0.2$ & $-0.1 \pm 0.0$ & $0.1 \pm 0.0$ & $0.0 \pm 0.0$ & $1.1 \pm 0.1$ & $0.0 \pm 0.0$ & $1.1 \pm 0.2$ \\
$\mathrm{~K} 409 \mathrm{~A}$ & $0.9 \pm 0.5$ & $2.3 \pm 0.1$ & $-2.3 \pm 0.1$ & $0.3 \pm 0.0$ & $1.2 \pm 0.5$ & $-0.3 \pm 0.2$ & $0.9 \pm 0.7$ \\
$\mathrm{E} 98^{\prime} \mathrm{A}$ & $1.2 \pm 0.2$ & $0.2 \pm 0.1$ & $-0.6 \pm 0.1$ & $0.2 \pm 0.0$ & $1.0 \pm 0.2$ & $0.0 \pm 0.0$ & $0.9 \pm 0.2$ \\
I183A & $1.1 \pm 0.1$ & $-0.1 \pm 0.0$ & $-0.1 \pm 0.0$ & $0.1 \pm 0.0$ & $1.0 \pm 0.1$ & $-0.1 \pm 0.0$ & $0.9 \pm 0.1$ \\
N408A & $1.0 \pm 0.2$ & $0.1 \pm 0.1$ & $-0.3 \pm 0.0$ & $0.0 \pm 0.0$ & $0.8 \pm 0.3$ & $-0.1 \pm 0.1$ & $0.6 \pm 0.4$ \\
S226A & $0.5 \pm 0.0$ & $0.2 \pm 0.2$ & $-0.1 \pm 0.0$ & $0.0 \pm 0.0$ & $0.6 \pm 0.1$ & $-0.1 \pm 0.0$ & $0.5 \pm 0.1$ \\
F320'A & $0.6 \pm 0.1$ & $0.0 \pm 0.0$ & $-0.2 \pm 0.0$ & $0.0 \pm 0.0$ & $0.5 \pm 0.1$ & $0.0 \pm 0.0$ & $0.5 \pm 0.1$ \\
E397A & $0.2 \pm 0.0$ & $-1.1 \pm 0.1$ & $1.2 \pm 0.1$ & $0.0 \pm 0.0$ & $0.3 \pm 0.1$ & $0.0 \pm 0.0$ & $0.3 \pm 0.1$ \\
F317'A & $0.4 \pm 0.1$ & $0.1 \pm 0.0$ & $-0.2 \pm 0.1$ & $0.0 \pm 0.0$ & $0.3 \pm 0.1$ & $-0.1 \pm 0.0$ & $0.3 \pm 0.1$ \\
T411A & $0.3 \pm 0.1$ & $-0.5 \pm 0.2$ & $0.3 \pm 0.1$ & $0.0 \pm 0.0$ & $0.2 \pm 0.0$ & $0.0 \pm 0.0$ & $0.2 \pm 0.1$ \\
I74A & $0.1 \pm 0.0$ & $0.0 \pm 0.0$ & $0.1 \pm 0.0$ & $0.0 \pm 0.0$ & $0.2 \pm 0.0$ & $0.0 \pm 0.0$ & $0.2 \pm 0.0$ \\
H254A & $0.2 \pm 0.0$ & $0.0 \pm 0.0$ & $0.0 \pm 0.0$ & $0.0 \pm 0.0$ & $0.2 \pm 0.0$ & $0.0 \pm 0.0$ & $0.2 \pm 0.0$ \\
\hline Total & $22.8 \pm 1.0$ & $1.6 \pm 0.2$ & $-3.8 \pm 0.2$ & $1.8 \pm 0.1$ & $22.5 \pm 1.0$ & $-2.4 \pm 0.1$ & $20.1 \pm 1.1$ \\
\hline
\end{tabular}


Table S5. Same as Table S1 but for compound 4.

\begin{tabular}{cccccccc}
\hline Residue & $\Delta \Delta \mathrm{E}_{\mathrm{vdw}}$ & $\Delta \Delta \mathrm{E}_{\text {ele }}$ & $\Delta \Delta \mathrm{GB}$ & $\Delta \Delta \mathrm{NP}$ & $\Delta \Delta \mathrm{H}$ & $\mathrm{IE}$ & $\Delta \Delta G_{\text {bind }}^{x \rightarrow a}$ \\
\hline Y105'A & $6.4 \pm 0.8$ & $1.1 \pm 1.4$ & $-1.5 \pm 0.8$ & $0.6 \pm 0.1$ & $6.6 \pm 0.9$ & $-0.9 \pm 0.5$ & $5.7 \pm 0.5$ \\
L166A & $3.3 \pm 0.2$ & $0.0 \pm 0.0$ & $-0.2 \pm 0.0$ & $0.2 \pm 0.0$ & $3.4 \pm 0.2$ & $-0.5 \pm 0.0$ & $2.9 \pm 0.3$ \\
$\mathrm{R} 425 \mathrm{~A}$ & $2.2 \pm 0.2$ & $-0.5 \pm 0.1$ & $0.4 \pm 0.0$ & $0.1 \pm 0.0$ & $2.2 \pm 0.2$ & $-0.1 \pm 0.0$ & $2.2 \pm 0.2$ \\
$\mathrm{~L} 172 \mathrm{~A}$ & $1.9 \pm 0.1$ & $0.0 \pm 0.0$ & $-0.1 \pm 0.0$ & $0.1 \pm 0.0$ & $1.9 \pm 0.2$ & $-0.1 \pm 0.1$ & $1.8 \pm 0.1$ \\
$\mathrm{Y} 176 \mathrm{~A}$ & $1.6 \pm 0.2$ & $-0.1 \pm 0.1$ & $-0.2 \pm 0.1$ & $0.1 \pm 0.0$ & $1.5 \pm 0.1$ & $-0.1 \pm 0.0$ & $1.4 \pm 0.1$ \\
$\mathrm{~N} 410 \mathrm{~A}$ & $1.5 \pm 0.1$ & $0.8 \pm 0.1$ & $-0.8 \pm 0.1$ & $0.1 \pm 0.0$ & $1.6 \pm 0.1$ & $-0.3 \pm 0.1$ & $1.3 \pm 0.1$ \\
$\mathrm{I} 183 \mathrm{~A}$ & $1.6 \pm 0.8$ & $0.0 \pm 0.1$ & $-0.5 \pm 0.4$ & $0.2 \pm 0.1$ & $1.2 \pm 0.6$ & $-0.1 \pm 0.1$ & $1.1 \pm 0.5$ \\
$\mathrm{H} 171 \mathrm{~A}$ & $1.0 \pm 0.1$ & $0.0 \pm 0.0$ & $0.1 \pm 0.1$ & $0.0 \pm 0.0$ & $1.1 \pm 0.1$ & $0.0 \pm 0.1$ & $1.1 \pm 0.2$ \\
$\mathrm{~K} 409 \mathrm{~A}$ & $1.0 \pm 0.1$ & $2.0 \pm 0.1$ & $-2.1 \pm 0.1$ & $0.3 \pm 0.0$ & $1.2 \pm 0.1$ & $-0.4 \pm 0.3$ & $0.9 \pm 0.4$ \\
$\mathrm{E} 98^{\prime} \mathrm{A}$ & $1.2 \pm 0.1$ & $0.2 \pm 0.1$ & $-0.6 \pm 0.1$ & $0.2 \pm 0.0$ & $0.9 \pm 0.1$ & $-0.1 \pm 0.0$ & $0.8 \pm 0.0$ \\
$\mathrm{~F} 320 \mathrm{~A}$ & $0.7 \pm 0.1$ & $0.0 \pm 0.1$ & $-0.2 \pm 0.1$ & $0.0 \pm 0.0$ & $0.5 \pm 0.2$ & $0.0 \pm 0.0$ & $0.5 \pm 0.2$ \\
$\mathrm{~F} 317{ }^{\prime} \mathrm{A}$ & $1.3 \pm 0.7$ & $0.2 \pm 0.2$ & $-0.9 \pm 0.4$ & $0.1 \pm 0.1$ & $0.7 \pm 0.4$ & $-0.2 \pm 0.2$ & $0.5 \pm 0.3$ \\
N408A & $0.9 \pm 0.1$ & $0.0 \pm 0.1$ & $-0.3 \pm 0.0$ & $0.1 \pm 0.0$ & $0.7 \pm 0.2$ & $-0.2 \pm 0.1$ & $0.5 \pm 0.3$ \\
S226A & $0.5 \pm 0.0$ & $0.1 \pm 0.1$ & $-0.1 \pm 0.0$ & $0.0 \pm 0.0$ & $0.5 \pm 0.1$ & $-0.1 \pm 0.1$ & $0.4 \pm 0.1$ \\
E397A & $0.2 \pm 0.0$ & $-1.0 \pm 0.1$ & $1.0 \pm 0.1$ & $0.0 \pm 0.0$ & $0.3 \pm 0.0$ & $0.0 \pm 0.0$ & $0.3 \pm 0.0$ \\
K181A & $0.3 \pm 0.4$ & $0.5 \pm 0.1$ & $-0.5 \pm 0.1$ & $0.0 \pm 0.0$ & $0.3 \pm 0.0$ & $0.0 \pm 0.0$ & $0.3 \pm 0.4$ \\
T411A & $0.4 \pm 0.0$ & $-0.5 \pm 0.1$ & $0.3 \pm 0.1$ & $0.0 \pm 0.0$ & $0.2 \pm 0.0$ & $0.0 \pm 0.0$ & $0.2 \pm 0.0$ \\
I74A & $0.1 \pm 0.0$ & $0.0 \pm 0.0$ & $0.1 \pm 0.0$ & $0.0 \pm 0.0$ & $0.2 \pm 0.0$ & $0.0 \pm 0.0$ & $0.2 \pm 0.0$ \\
H254A & $0.2 \pm 0.0$ & $0.0 \pm 0.0$ & $0.0 \pm 0.0$ & $0.0 \pm 0.0$ & $0.2 \pm 0.0$ & $0.0 \pm 0.0$ & $0.2 \pm 0.0$ \\
D168A & $0.0 \pm 0.0$ & $0.8 \pm 0.1$ & $-0.7 \pm 0.1$ & $0.0 \pm 0.0$ & $0.2 \pm 0.0$ & $0.0 \pm 0.0$ & $0.2 \pm 0.0$ \\
\hline Total & $26.3 \pm 1.4$ & $3.5 \pm 1.2$ & $-6.3 \pm 0.9$ & $2.1 \pm 0.1$ & $25.5 \pm 0.9$ & $-2.9 \pm 0.6$ & $22.7 \pm 1.2$ \\
\hline
\end{tabular}


Table S6. Same as Table S1 but for compound 5.

\begin{tabular}{|c|c|c|c|c|c|c|c|}
\hline Residue & $\Delta \Delta \mathrm{E}_{\mathrm{vdw}}$ & $\Delta \Delta \mathrm{E}_{\text {ele }}$ & $\Delta \Delta \mathrm{GB}$ & $\Delta \Delta \mathrm{NP}$ & $\Delta \Delta \mathrm{H}$ & $\mathrm{IE}$ & $\Delta \Delta G_{\text {bind }}^{x \rightarrow a}$ \\
\hline Y105'A & $5.3 \pm 0.2$ & $0.3 \pm 0.1$ & $-0.8 \pm 0.1$ & $0.5 \pm 0.0$ & $5.2 \pm 0.1$ & $-0.6 \pm 0.1$ & $4.6 \pm 0.1$ \\
\hline L166A & $3.2 \pm 0.1$ & $0.1 \pm 0.1$ & $-0.2 \pm 0.0$ & $0.2 \pm 0.0$ & $3.3 \pm 0.1$ & $-0.7 \pm 0.1$ & $2.6 \pm 0.3$ \\
\hline $\mathrm{R} 425 \mathrm{~A}$ & $2.2 \pm 0.1$ & $-0.5 \pm 0.0$ & $0.4 \pm 0.0$ & $0.1 \pm 0.0$ & $2.2 \pm 0.1$ & $-0.1 \pm 0.0$ & $2.1 \pm 0.1$ \\
\hline L172A & $1.7 \pm 0.1$ & $0.0 \pm 0.0$ & $-0.1 \pm 0.0$ & $0.1 \pm 0.0$ & $1.7 \pm 0.1$ & $-0.1 \pm 0.0$ & $1.6 \pm 0.1$ \\
\hline N410A & $1.4 \pm 0.1$ & $0.9 \pm 0.3$ & $-0.8 \pm 0.1$ & $0.1 \pm 0.0$ & $1.6 \pm 0.1$ & $-0.4 \pm 0.0$ & $1.3 \pm 0.1$ \\
\hline Y176A & $1.5 \pm 0.2$ & $0.0 \pm 0.3$ & $-0.2 \pm 0.2$ & $0.2 \pm 0.0$ & $1.4 \pm 0.1$ & $-0.3 \pm 0.1$ & $1.2 \pm 0.1$ \\
\hline H171A & $1.0 \pm 0.0$ & $-0.1 \pm 0.0$ & $0.1 \pm 0.0$ & $0.0 \pm 0.0$ & $1.1 \pm 0.0$ & $0.0 \pm 0.0$ & $1.1 \pm 0.1$ \\
\hline E98'A & $1.1 \pm 0.1$ & $0.3 \pm 0.1$ & $-0.7 \pm 0.0$ & $0.2 \pm 0.0$ & $0.9 \pm 0.1$ & $-0.1 \pm 0.0$ & $0.8 \pm 0.0$ \\
\hline K409A & $0.9 \pm 0.2$ & $2.2 \pm 0.1$ & $-2.2 \pm 0.1$ & $0.3 \pm 0.0$ & $1.1 \pm 0.2$ & $-0.3 \pm 0.1$ & $0.8 \pm 0.3$ \\
\hline $\mathrm{I} 183 \mathrm{~A}$ & $1.1 \pm 0.1$ & $-0.1 \pm 0.0$ & $-0.4 \pm 0.2$ & $0.1 \pm 0.0$ & $0.8 \pm 0.1$ & $-0.2 \pm 0.1$ & $0.6 \pm 0.2$ \\
\hline N408A & $1.0 \pm 0.1$ & $0.0 \pm 0.0$ & $-0.3 \pm 0.0$ & $0.1 \pm 0.0$ & $0.7 \pm 0.1$ & $-0.2 \pm 0.1$ & $0.6 \pm 0.2$ \\
\hline F320'A & $0.6 \pm 0.0$ & $0.0 \pm 0.1$ & $-0.1 \pm 0.1$ & $0.0 \pm 0.0$ & $0.4 \pm 0.0$ & $0.0 \pm 0.0$ & $0.4 \pm 0.0$ \\
\hline S226A & $0.5 \pm 0.0$ & $0.1 \pm 0.0$ & $-0.1 \pm 0.0$ & $0.0 \pm 0.0$ & $0.5 \pm 0.0$ & $-0.1 \pm 0.0$ & $0.4 \pm 0.0$ \\
\hline E397A & $0.2 \pm 0.0$ & $-1.0 \pm 0.1$ & $1.1 \pm 0.0$ & $0.0 \pm 0.0$ & $0.3 \pm 0.1$ & $-0.0 \pm 0.0$ & $0.3 \pm 0.0$ \\
\hline T411A & $0.4 \pm 0.0$ & $-0.6 \pm 0.0$ & $0.4 \pm 0.0$ & $0.0 \pm 0.0$ & $0.2 \pm 0.0$ & $0.0 \pm 0.0$ & $0.2 \pm 0.0$ \\
\hline $\mathrm{I} 74 \mathrm{~A}$ & $0.1 \pm 0.0$ & $0.0 \pm 0.0$ & $0.1 \pm 0.0$ & $0.0 \pm 0.0$ & $0.2 \pm 0.0$ & $0.0 \pm 0.0$ & $0.2 \pm 0.0$ \\
\hline Total & $22.1 \pm 0.3$ & $1.8 \pm 0.3$ & $-3.9 \pm 0.3$ & $1.8 \pm 0.1$ & $21.7 \pm 0.3$ & $-3.0 \pm 0.2$ & $18.7 \pm 0.4$ \\
\hline
\end{tabular}


Table S7. Same as Table S1 but for compound 6.

\begin{tabular}{cccccccc}
\hline Residue & $\Delta \Delta \mathrm{E}_{\mathrm{vdw}}$ & $\Delta \Delta \mathrm{E}_{\text {ele }}$ & $\Delta \Delta \mathrm{GB}$ & $\Delta \Delta \mathrm{NP}$ & $\Delta \Delta \mathrm{H}$ & $\mathrm{IE}$ & $\Delta \Delta G_{\text {bind }}^{x \rightarrow a}$ \\
\hline Y105'A & $5.5 \pm 0.2$ & $0.0 \pm 0.0$ & $-0.7 \pm 0.0$ & $0.5 \pm 0.0$ & $5.3 \pm 0.3$ & $-0.7 \pm 0.1$ & $4.6 \pm 0.4$ \\
L166A & $3.0 \pm 0.3$ & $0.0 \pm 0.0$ & $-0.3 \pm 0.0$ & $0.2 \pm 0.0$ & $2.9 \pm 0.3$ & $-0.7 \pm 0.2$ & $2.3 \pm 0.5$ \\
L172A & $2.1 \pm 0.1$ & $0.0 \pm 0.0$ & $-0.1 \pm 0.0$ & $0.1 \pm 0.0$ & $2.1 \pm 0.1$ & $-0.2 \pm 0.0$ & $1.9 \pm 0.1$ \\
$\mathrm{R} 425 \mathrm{~A}$ & $1.7 \pm 0.0$ & $-0.3 \pm 0.0$ & $0.3 \pm 0.0$ & $0.1 \pm 0.0$ & $1.8 \pm 0.0$ & $0.0 \pm 0.0$ & $1.7 \pm 0.0$ \\
$\mathrm{H} 171 \mathrm{~A}$ & $1.3 \pm 0.0$ & $-0.2 \pm 0.0$ & $0.2 \pm 0.0$ & $0.0 \pm 0.0$ & $1.3 \pm 0.0$ & $0.1 \pm 0.0$ & $1.3 \pm 0.0$ \\
$\mathrm{E} 98^{\prime} \mathrm{A}$ & $1.4 \pm 0.0$ & $0.4 \pm 0.0$ & $-0.9 \pm 0.0$ & $0.2 \pm 0.0$ & $1.2 \pm 0.0$ & $-0.1 \pm 0.0$ & $1.1 \pm 0.0$ \\
N410A & $1.1 \pm 0.1$ & $0.4 \pm 0.0$ & $-0.5 \pm 0.0$ & $0.1 \pm 0.0$ & $1.2 \pm 0.1$ & $-0.2 \pm 0.0$ & $1.0 \pm 0.1$ \\
Y176A & $1.3 \pm 0.1$ & $0.0 \pm 0.1$ & $-0.2 \pm 0.1$ & $0.1 \pm 0.0$ & $1.2 \pm 0.1$ & $-0.2 \pm 0.1$ & $1.0 \pm 0.2$ \\
N408A & $1.1 \pm 0.0$ & $0.1 \pm 0.0$ & $-0.3 \pm 0.0$ & $0.1 \pm 0.0$ & $1.0 \pm 0.1$ & $0.0 \pm 0.0$ & $0.9 \pm 0.1$ \\
K409A & $0.4 \pm 0.2$ & $3.2 \pm 0.1$ & $-2.7 \pm 0.0$ & $0.2 \pm 0.0$ & $1.0 \pm 0.2$ & $-0.3 \pm 0.1$ & $0.7 \pm 0.3$ \\
F320'A & $0.8 \pm 0.0$ & $0.0 \pm 0.0$ & $-0.2 \pm 0.0$ & $0.0 \pm 0.0$ & $0.6 \pm 0.0$ & $0.0 \pm 0.0$ & $0.6 \pm 0.0$ \\
S226A & $0.5 \pm 0.0$ & $0.4 \pm 0.1$ & $-0.2 \pm 0.0$ & $0.0 \pm 0.0$ & $0.7 \pm 0.1$ & $-0.1 \pm 0.0$ & $0.6 \pm 0.1$ \\
I183A & $1.0 \pm 0.1$ & $-0.1 \pm 0.0$ & $-0.2 \pm 0.1$ & $0.1 \pm 0.0$ & $0.8 \pm 0.0$ & $-0.3 \pm 0.1$ & $0.6 \pm 0.1$ \\
F317'A & $0.6 \pm 0.1$ & $0.1 \pm 0.0$ & $-0.2 \pm 0.1$ & $0.1 \pm 0.0$ & $0.5 \pm 0.0$ & $-0.1 \pm 0.1$ & $0.4 \pm 0.0$ \\
I74A & $0.1 \pm 0.0$ & $0.0 \pm 0.0$ & $0.1 \pm 0.0$ & $0.0 \pm 0.0$ & $0.2 \pm 0.0$ & $0.0 \pm 0.0$ & $0.2 \pm 0.0$ \\
H254A & $0.2 \pm 0.0$ & $0.0 \pm 0.0$ & $0.0 \pm 0.0$ & $0.0 \pm 0.0$ & $0.2 \pm 0.0$ & $0.0 \pm 0.0$ & $0.2 \pm 0.0$ \\
T411A & $0.2 \pm 0.0$ & $-0.2 \pm 0.1$ & $0.1 \pm 0.1$ & $0.0 \pm 0.0$ & $0.2 \pm 0.0$ & $0.0 \pm 0.0$ & $0.2 \pm 0.0$ \\
\hline Total & $22.3 \pm 0.4$ & $3.8 \pm 0.0$ & $-5.8 \pm 0.2$ & $1.8 \pm 0.0$ & $22.1 \pm 0.4$ & $-2.9 \pm 0.2$ & $19.2 \pm 0.6$ \\
\hline
\end{tabular}


Table S8. Same as Table S1 but for compound 7.

\begin{tabular}{cccccccc}
\hline Residue & $\Delta \Delta \mathrm{E}_{\mathrm{vdw}}$ & $\Delta \Delta \mathrm{E}_{\text {ele }}$ & $\Delta \Delta \mathrm{GB}$ & $\Delta \Delta \mathrm{NP}$ & $\Delta \Delta \mathrm{H}$ & $\mathrm{IE}$ & $\Delta \Delta G_{\text {bind }}^{x \rightarrow a}$ \\
\hline Y105'A & $6.5 \pm 0.8$ & $0.6 \pm 0.4$ & $-1.3 \pm 0.4$ & $0.5 \pm 0.1$ & $6.4 \pm 0.8$ & $-0.6 \pm 0.3$ & $5.9 \pm 0.5$ \\
L166A & $3.4 \pm 0.2$ & $0.0 \pm 0.1$ & $-0.2 \pm 0.1$ & $0.2 \pm 0.0$ & $3.5 \pm 0.2$ & $-0.5 \pm 0.1$ & $3.0 \pm 0.3$ \\
$\mathrm{R} 425 \mathrm{~A}$ & $2.0 \pm 0.3$ & $-0.4 \pm 0.1$ & $0.4 \pm 0.1$ & $0.1 \pm 0.0$ & $2.0 \pm 0.3$ & $-0.1 \pm 0.0$ & $1.9 \pm 0.2$ \\
$\mathrm{~L} 172 \mathrm{~A}$ & $2.0 \pm 0.1$ & $0.0 \pm 0.0$ & $-0.1 \pm 0.0$ & $0.1 \pm 0.0$ & $2.0 \pm 0.1$ & $-0.2 \pm 0.1$ & $1.8 \pm 0.1$ \\
$\mathrm{Y} 176 \mathrm{~A}$ & $1.6 \pm 0.1$ & $0.8 \pm 1.4$ & $-0.7 \pm 0.9$ & $0.2 \pm 0.1$ & $1.8 \pm 0.5$ & $-0.3 \pm 0.4$ & $1.5 \pm 0.1$ \\
$\mathrm{H} 171 \mathrm{~A}$ & $1.2 \pm 0.1$ & $-0.2 \pm 0.1$ & $0.2 \pm 0.1$ & $0.0 \pm 0.0$ & $1.2 \pm 0.1$ & $0.0 \pm 0.0$ & $1.2 \pm 0.1$ \\
$\mathrm{~K} 409 \mathrm{~A}$ & $1.1 \pm 0.2$ & $1.8 \pm 0.2$ & $-1.8 \pm 0.2$ & $0.3 \pm 0.0$ & $1.4 \pm 0.2$ & $-0.2 \pm 0.1$ & $1.2 \pm 0.3$ \\
N410A & $1.3 \pm 0.1$ & $0.5 \pm 0.1$ & $-0.6 \pm 0.1$ & $0.1 \pm 0.0$ & $1.4 \pm 0.1$ & $-0.2 \pm 0.1$ & $1.1 \pm 0.0$ \\
E98'A & $1.3 \pm 0.2$ & $0.4 \pm 0.1$ & $-0.8 \pm 0.0$ & $0.2 \pm 0.0$ & $1.0 \pm 0.1$ & $-0.1 \pm 0.0$ & $1.0 \pm 0.1$ \\
N408A & $1.1 \pm 0.0$ & $0.1 \pm 0.1$ & $-0.3 \pm 0.0$ & $0.1 \pm 0.0$ & $1.0 \pm 0.1$ & $-0.1 \pm 0.0$ & $0.9 \pm 0.1$ \\
I183A & $0.9 \pm 0.2$ & $-0.1 \pm 0.0$ & $-0.1 \pm 0.0$ & $0.1 \pm 0.0$ & $0.9 \pm 0.2$ & $-0.1 \pm 0.1$ & $0.7 \pm 0.3$ \\
S226A & $0.5 \pm 0.0$ & $0.4 \pm 0.2$ & $-0.2 \pm 0.0$ & $0.0 \pm 0.0$ & $0.7 \pm 0.2$ & $-0.1 \pm 0.0$ & $0.6 \pm 0.2$ \\
F320'A & $0.7 \pm 0.1$ & $0.0 \pm 0.1$ & $-0.2 \pm 0.1$ & $0.0 \pm 0.0$ & $0.5 \pm 0.1$ & $0.0 \pm 0.0$ & $0.5 \pm 0.1$ \\
E397A & $0.2 \pm 0.0$ & $-0.8 \pm 0.1$ & $0.9 \pm 0.0$ & $0.0 \pm 0.0$ & $0.3 \pm 0.1$ & $0.0 \pm 0.0$ & $0.3 \pm 0.1$ \\
F317'A & $0.4 \pm 0.1$ & $0.1 \pm 0.1$ & $-0.2 \pm 0.2$ & $0.0 \pm 0.0$ & $0.3 \pm 0.1$ & $0.0 \pm 0.0$ & $0.3 \pm 0.1$ \\
I74A & $0.1 \pm 0.0$ & $0.0 \pm 0.0$ & $0.1 \pm 0.0$ & $0.0 \pm 0.0$ & $0.2 \pm 0.0$ & $0.0 \pm 0.0$ & $0.2 \pm 0.0$ \\
T411A & $0.3 \pm 0.1$ & $-0.3 \pm 0.1$ & $0.1 \pm 0.1$ & $0.0 \pm 0.0$ & $0.2 \pm 0.1$ & $0.0 \pm 0.0$ & $0.2 \pm 0.1$ \\
H254A & $0.2 \pm 0.0$ & $0.0 \pm 0.0$ & $0.0 \pm 0.0$ & $0.0 \pm 0.0$ & $0.2 \pm 0.0$ & $0.0 \pm 0.0$ & $0.2 \pm 0.0$ \\
\hline Total & $24.8 \pm 0.2$ & $2.8 \pm 0.3$ & $-4.7 \pm 0.2$ & $1.9 \pm 0.0$ & $24.9 \pm 0.2$ & $-2.4 \pm 0.1$ & $22.5 \pm 0.1$ \\
\hline
\end{tabular}


Table S9. Same as Table S1 but for compound 8.

\begin{tabular}{|c|c|c|c|c|c|c|c|}
\hline Residue & $\Delta \Delta \mathrm{E}_{\mathrm{vdw}}$ & $\Delta \Delta \mathrm{E}_{\text {ele }}$ & $\Delta \Delta \mathrm{GB}$ & $\Delta \Delta \mathrm{NP}$ & $\Delta \Delta \mathrm{H}$ & IE & $\Delta \Delta G_{\text {bind }}^{x \rightarrow a}$ \\
\hline Y105'A & $5.2 \pm 0.0$ & $0.1 \pm 0.1$ & $-0.6 \pm 0.0$ & $0.5 \pm 0.0$ & $5.1 \pm 0.1$ & $-0.4 \pm 0.1$ & $4.6 \pm 0.2$ \\
\hline L166A & $3.2 \pm 0.0$ & $0.1 \pm 0.0$ & $-0.2 \pm 0.0$ & $0.2 \pm 0.0$ & $3.3 \pm 0.1$ & $-0.5 \pm 0.2$ & $2.8 \pm 0.2$ \\
\hline $\mathrm{R} 425 \mathrm{~A}$ & $2.3 \pm 0.0$ & $-0.5 \pm 0.0$ & $0.3 \pm 0.1$ & $0.1 \pm 0.0$ & $2.2 \pm 0.0$ & $-0.1 \pm 0.0$ & $2.1 \pm 0.0$ \\
\hline L172A & $1.8 \pm 0.0$ & $0.0 \pm 0.0$ & $-0.1 \pm 0.0$ & $0.1 \pm 0.0$ & $1.8 \pm 0.1$ & $-0.1 \pm 0.0$ & $1.7 \pm 0.0$ \\
\hline N410A & $1.5 \pm 0.0$ & $1.0 \pm 0.2$ & $-0.8 \pm 0.1$ & $0.1 \pm 0.0$ & $1.7 \pm 0.0$ & $-0.3 \pm 0.0$ & $1.4 \pm 0.0$ \\
\hline Y176A & $1.4 \pm 0.0$ & $0.1 \pm 0.1$ & $-0.2 \pm 0.0$ & $0.1 \pm 0.0$ & $1.4 \pm 0.0$ & $-0.1 \pm 0.0$ & $1.3 \pm 0.0$ \\
\hline H171A & $1.0 \pm 0.0$ & $-0.1 \pm 0.1$ & $0.1 \pm 0.1$ & $0.0 \pm 0.0$ & $1.1 \pm 0.0$ & $0.0 \pm 0.0$ & $1.1 \pm 0.0$ \\
\hline E98'A & $1.0 \pm 0.1$ & $0.3 \pm 0.0$ & $-0.6 \pm 0.0$ & $0.1 \pm 0.0$ & $0.9 \pm 0.0$ & $0.0 \pm 0.0$ & $0.8 \pm 0.0$ \\
\hline K409A & $0.9 \pm 0.1$ & $2.3 \pm 0.0$ & $-2.3 \pm 0.1$ & $0.3 \pm 0.0$ & $1.2 \pm 0.1$ & $-0.4 \pm 0.1$ & $0.8 \pm 0.2$ \\
\hline $\mathrm{I} 183 \mathrm{~A}$ & $0.9 \pm 0.1$ & $0.0 \pm 0.0$ & $-0.4 \pm 0.1$ & $0.1 \pm 0.0$ & $0.6 \pm 0.1$ & $-0.1 \pm 0.0$ & $0.5 \pm 0.1$ \\
\hline S226A & $0.5 \pm 0.0$ & $0.1 \pm 0.0$ & $-0.1 \pm 0.0$ & $0.0 \pm 0.0$ & $0.5 \pm 0.0$ & $-0.1 \pm 0.0$ & $0.4 \pm 0.0$ \\
\hline F320'A & $0.6 \pm 0.0$ & $0.0 \pm 0.0$ & $-0.2 \pm 0.0$ & $0.0 \pm 0.0$ & $0.4 \pm 0.0$ & $0.0 \pm 0.0$ & $0.4 \pm 0.0$ \\
\hline N408A & $0.9 \pm 0.1$ & $0.0 \pm 0.0$ & $-0.4 \pm 0.1$ & $0.0 \pm 0.0$ & $0.6 \pm 0.1$ & $-0.3 \pm 0.0$ & $0.4 \pm 0.1$ \\
\hline E397A & $0.2 \pm 0.0$ & $-1.1 \pm 0.0$ & $1.1 \pm 0.0$ & $0.0 \pm 0.0$ & $0.3 \pm 0.0$ & $0.0 \pm 0.0$ & $0.3 \pm 0.0$ \\
\hline T411A & $0.4 \pm 0.0$ & $-0.6 \pm 0.0$ & $0.4 \pm 0.0$ & $0.0 \pm 0.0$ & $0.2 \pm 0.0$ & $0.0 \pm 0.0$ & $0.2 \pm 0.0$ \\
\hline $\mathrm{I} 74 \mathrm{~A}$ & $0.1 \pm 0.0$ & $0.0 \pm 0.0$ & $0.1 \pm 0.0$ & $0.0 \pm 0.0$ & $0.2 \pm 0.0$ & $0.0 \pm 0.0$ & $0.2 \pm 0.0$ \\
\hline F317'A & $0.3 \pm 0.0$ & $0.0 \pm 0.0$ & $-0.2 \pm 0.0$ & $0.0 \pm 0.0$ & $0.2 \pm 0.0$ & $0.0 \pm 0.0$ & $0.2 \pm 0.0$ \\
\hline $\mathrm{H} 254 \mathrm{~A}$ & $0.2 \pm 0.0$ & $0.0 \pm 0.0$ & $0.0 \pm 0.0$ & $0.0 \pm 0.0$ & $0.2 \pm 0.0$ & $0.0 \pm 0.0$ & $0.2 \pm 0.0$ \\
\hline D168A & $0.0 \pm 0.0$ & $0.7 \pm 0.0$ & $-0.6 \pm 0.0$ & $0.0 \pm 0.0$ & $0.2 \pm 0.0$ & $0.0 \pm 0.0$ & $0.2 \pm 0.0$ \\
\hline Total & $22.4 \pm 0.2$ & $2.5 \pm 0.4$ & $-4.7 \pm 0.2$ & $1.7 \pm 0.1$ & $22.0 \pm 0.1$ & $-2.5 \pm 0.3$ & $19.5 \pm 0.2$ \\
\hline
\end{tabular}


Table S10. Same as Table S1 but for compound 9.

\begin{tabular}{|c|c|c|c|c|c|c|c|}
\hline Residue & $\Delta \Delta \mathrm{E}_{\mathrm{vdw}}$ & $\Delta \Delta \mathrm{E}_{\text {ele }}$ & $\Delta \Delta \mathrm{GB}$ & $\Delta \Delta \mathrm{NP}$ & $\Delta \Delta \mathrm{H}$ & IE & $\Delta \Delta G_{\text {bind }}^{x \rightarrow a}$ \\
\hline Y105'A & $5.5 \pm 0.2$ & $0.1 \pm 0.0$ & $-0.7 \pm 0.0$ & $0.5 \pm 0.0$ & $5.4 \pm 0.2$ & $-0.4 \pm 0.1$ & $5.0 \pm 0.2$ \\
\hline L166A & $3.3 \pm 0.2$ & $0.1 \pm 0.0$ & $-0.2 \pm 0.0$ & $0.2 \pm 0.0$ & $3.4 \pm 0.2$ & $-0.5 \pm 0.1$ & $2.9 \pm 0.2$ \\
\hline R425A & $2.1 \pm 0.2$ & $-0.4 \pm 0.1$ & $0.3 \pm 0.1$ & $0.1 \pm 0.0$ & $2.1 \pm 0.2$ & $-0.1 \pm 0.0$ & $2.0 \pm 0.2$ \\
\hline L172A & $1.8 \pm 0.1$ & $0.0 \pm 0.0$ & $-0.1 \pm 0.0$ & $0.1 \pm 0.0$ & $1.9 \pm 0.1$ & $-0.1 \pm 0.1$ & $1.7 \pm 0.0$ \\
\hline Y176A & $1.6 \pm 0.1$ & $-0.1 \pm 0.0$ & $-0.2 \pm 0.0$ & $0.1 \pm 0.0$ & $1.6 \pm 0.1$ & $-0.1 \pm 0.1$ & $1.5 \pm 0.1$ \\
\hline N410A & $1.4 \pm 0.1$ & $0.7 \pm 0.3$ & $-0.7 \pm 0.2$ & $0.1 \pm 0.0$ & $1.5 \pm 0.2$ & $-0.3 \pm 0.1$ & $1.2 \pm 0.2$ \\
\hline H171A & $1.1 \pm 0.1$ & $0.0 \pm 0.0$ & $0.0 \pm 0.0$ & $0.0 \pm 0.0$ & $1.1 \pm 0.1$ & $0.0 \pm 0.0$ & $1.2 \pm 0.1$ \\
\hline I183A & $1.6 \pm 0.1$ & $0.0 \pm 0.0$ & $-0.5 \pm 0.1$ & $0.2 \pm 0.0$ & $1.3 \pm 0.1$ & $-0.2 \pm 0.0$ & $1.1 \pm 0.1$ \\
\hline E98'A & $1.2 \pm 0.1$ & $0.2 \pm 0.0$ & $-0.6 \pm 0.1$ & $0.2 \pm 0.0$ & $0.9 \pm 0.1$ & $-0.1 \pm 0.1$ & $0.9 \pm 0.1$ \\
\hline K409A & $0.9 \pm 0.2$ & $2.2 \pm 0.1$ & $-2.2 \pm 0.1$ & $0.3 \pm 0.0$ & $1.1 \pm 0.2$ & $-0.3 \pm 0.2$ & $0.8 \pm 0.4$ \\
\hline N408A & $1.0 \pm 0.1$ & $0.1 \pm 0.1$ & $-0.4 \pm 0.0$ & $0.1 \pm 0.0$ & $0.8 \pm 0.2$ & $-0.2 \pm 0.1$ & $0.7 \pm 0.2$ \\
\hline S226A & $0.5 \pm 0.0$ & $0.3 \pm 0.2$ & $-0.1 \pm 0.0$ & $0.0 \pm 0.0$ & $0.6 \pm 0.1$ & $-0.1 \pm 0.0$ & $0.6 \pm 0.1$ \\
\hline F317'A & $1.0 \pm 0.1$ & $0.2 \pm 0.1$ & $-0.6 \pm 0.2$ & $0.1 \pm 0.0$ & $0.6 \pm 0.1$ & $-0.1 \pm 0.0$ & $0.6 \pm 0.1$ \\
\hline F320'A & $0.6 \pm 0.1$ & $0.0 \pm 0.0$ & $-0.1 \pm 0.0$ & $0.0 \pm 0.0$ & $0.5 \pm 0.1$ & $0.0 \pm 0.0$ & $0.5 \pm 0.1$ \\
\hline E397A & $0.2 \pm 0.0$ & $-1.1 \pm 0.1$ & $1.2 \pm 0.1$ & $0.0 \pm 0.0$ & $0.3 \pm 0.0$ & $0.0 \pm 0.0$ & $0.3 \pm 0.0$ \\
\hline I74A & $0.1 \pm 0.0$ & $0.0 \pm 0.0$ & $0.1 \pm 0.0$ & $0.0 \pm 0.0$ & $0.2 \pm 0.0$ & $0.0 \pm 0.0$ & $0.2 \pm 0.0$ \\
\hline T411A & $0.4 \pm 0.1$ & $-0.5 \pm 0.1$ & $0.3 \pm 0.1$ & $0.0 \pm 0.0$ & $0.2 \pm 0.0$ & $0.0 \pm 0.0$ & $0.2 \pm 0.0$ \\
\hline $\mathrm{H} 254 \mathrm{~A}$ & $0.2 \pm 0.0$ & $0.0 \pm 0.0$ & $0.0 \pm 0.0$ & $0.0 \pm 0.0$ & $0.2 \pm 0.0$ & $0.0 \pm 0.0$ & $0.2 \pm 0.0$ \\
\hline Total & $24.6 \pm 0.6$ & $1.7 \pm 0.1$ & $-4.4 \pm 0.2$ & $2.0 \pm 0.1$ & $23.8 \pm 0.5$ & $-2.4 \pm 0.3$ & $21.4 \pm 0.8$ \\
\hline
\end{tabular}


Table S11. Same as Table S1 but for compound 10.

\begin{tabular}{|c|c|c|c|c|c|c|c|}
\hline Residue & $\Delta \Delta \mathrm{E}_{\mathrm{vdw}}$ & $\Delta \Delta \mathrm{E}_{\text {ele }}$ & $\Delta \Delta \mathrm{GB}$ & $\Delta \Delta \mathrm{NP}$ & $\Delta \Delta \mathrm{H}$ & IE & $\Delta \Delta G_{\text {bind }}^{x \rightarrow a}$ \\
\hline Y105'A & $4.8 \pm 0.1$ & $0.7 \pm 0.0$ & $-1.1 \pm 0.0$ & $0.5 \pm 0.0$ & $4.8 \pm 0.1$ & $-0.3 \pm 0.0$ & $4.4 \pm 0.1$ \\
\hline L166A & $3.7 \pm 0.1$ & $0.1 \pm 0.0$ & $-0.5 \pm 0.0$ & $0.2 \pm 0.0$ & $3.4 \pm 0.0$ & $-0.6 \pm 0.0$ & $2.8 \pm 0.0$ \\
\hline $\mathrm{R} 425 \mathrm{~A}$ & $2.4 \pm 0.0$ & $-0.3 \pm 0.0$ & $0.2 \pm 0.0$ & $0.1 \pm 0.0$ & $2.4 \pm 0.0$ & $-0.1 \pm 0.0$ & $2.3 \pm 0.0$ \\
\hline N410A & $1.5 \pm 0.0$ & $0.9 \pm 0.0$ & $-0.7 \pm 0.0$ & $0.1 \pm 0.0$ & $1.8 \pm 0.0$ & $-0.4 \pm 0.0$ & $1.4 \pm 0.1$ \\
\hline L172A & $1.1 \pm 0.0$ & $0.1 \pm 0.0$ & $-0.1 \pm 0.0$ & $0.1 \pm 0.0$ & $1.1 \pm 0.0$ & $0.0 \pm 0.0$ & $1.1 \pm 0.0$ \\
\hline Y176A & $1.7 \pm 0.1$ & $0.0 \pm 0.0$ & $-0.4 \pm 0.0$ & $0.2 \pm 0.0$ & $1.4 \pm 0.1$ & $-0.4 \pm 0.0$ & $1.1 \pm 0.1$ \\
\hline H171A & $0.9 \pm 0.0$ & $0.1 \pm 0.0$ & $-0.1 \pm 0.0$ & $0.0 \pm 0.0$ & $1.0 \pm 0.0$ & $0.0 \pm 0.0$ & $0.9 \pm 0.0$ \\
\hline E98'A & $0.9 \pm 0.0$ & $0.2 \pm 0.1$ & $-0.5 \pm 0.1$ & $0.1 \pm 0.0$ & $0.7 \pm 0.0$ & $-0.1 \pm 0.0$ & $0.7 \pm 0.0$ \\
\hline I183A & $0.8 \pm 0.0$ & $0.0 \pm 0.0$ & $-0.2 \pm 0.0$ & $0.1 \pm 0.0$ & $0.7 \pm 0.0$ & $-0.1 \pm 0.0$ & $0.6 \pm 0.0$ \\
\hline S226A & $0.4 \pm 0.0$ & $0.2 \pm 0.1$ & $-0.1 \pm 0.0$ & $0.0 \pm 0.0$ & $0.5 \pm 0.1$ & $-0.1 \pm 0.0$ & $0.4 \pm 0.1$ \\
\hline N408A & $0.8 \pm 0.2$ & $0.1 \pm 0.0$ & $-0.4 \pm 0.0$ & $0.0 \pm 0.0$ & $0.5 \pm 0.2$ & $-0.3 \pm 0.0$ & $0.3 \pm 0.2$ \\
\hline E397A & $0.2 \pm 0.0$ & $-1.0 \pm 0.0$ & $1.0 \pm 0.0$ & $0.0 \pm 0.0$ & $0.3 \pm 0.0$ & $0.0 \pm 0.0$ & $0.3 \pm 0.0$ \\
\hline F320A & $0.4 \pm 0.0$ & $-0.1 \pm 0.0$ & $-0.1 \pm 0.0$ & $0.0 \pm 0.0$ & $0.2 \pm 0.0$ & $0.0 \pm 0.0$ & $0.2 \pm 0.0$ \\
\hline $\mathrm{I} 74 \mathrm{~A}$ & $0.1 \pm 0.0$ & $0.0 \pm 0.0$ & $0.1 \pm 0.0$ & $0.0 \pm 0.0$ & $0.2 \pm 0.0$ & $0.0 \pm 0.0$ & $0.2 \pm 0.0$ \\
\hline $\mathrm{T} 411 \mathrm{~A}$ & $0.4 \pm 0.0$ & $-0.5 \pm 0.0$ & $0.2 \pm 0.0$ & $0.0 \pm 0.0$ & $0.3 \pm 0.0$ & $0.0 \pm 0.0$ & $0.2 \pm 0.0$ \\
\hline $\mathrm{H} 254 \mathrm{~A}$ & $0.2 \pm 0.0$ & $0.0 \pm 0.0$ & $0.0 \pm 0.0$ & $0.0 \pm 0.0$ & $0.2 \pm 0.0$ & $0.0 \pm 0.0$ & $0.2 \pm 0.0$ \\
\hline F317'A & $0.3 \pm 0.0$ & $0.1 \pm 0.0$ & $-0.2 \pm 0.0$ & $0.0 \pm 0.0$ & $0.2 \pm 0.0$ & $-0.1 \pm 0.0$ & $0.2 \pm 0.0$ \\
\hline S417A & $0.3 \pm 0.0$ & $0.1 \pm 0.0$ & $-0.3 \pm 0.0$ & $0.1 \pm 0.0$ & $0.2 \pm 0.0$ & $0.0 \pm 0.0$ & $0.2 \pm 0.0$ \\
\hline Total & $20.9 \pm 0.6$ & $0.5 \pm 0.3$ & $-3.2 \pm 0.1$ & $1.6 \pm 0.1$ & $19.8 \pm 0.3$ & $-2.5 \pm 0.3$ & $17.3 \pm 0.0$ \\
\hline
\end{tabular}


Table S12. Same as Table S1 but for compound 11.

\begin{tabular}{cccccccc}
\hline Residue & $\Delta \Delta \mathrm{E}_{\mathrm{vdw}}$ & $\Delta \Delta \mathrm{E}_{\text {ele }}$ & $\Delta \Delta \mathrm{GB}$ & $\Delta \Delta \mathrm{NP}$ & $\Delta \Delta \mathrm{H}$ & $\mathrm{IE}$ & $\Delta \Delta G_{\text {bind }}^{x \rightarrow a}$ \\
\hline Y105'A & $4.2 \pm 0.1$ & $0.1 \pm 0.1$ & $-0.7 \pm 0.0$ & $0.4 \pm 0.0$ & $4.0 \pm 0.1$ & $-0.3 \pm 0.3$ & $3.7 \pm 0.3$ \\
L166A & $3.6 \pm 0.1$ & $0.1 \pm 0.0$ & $-0.6 \pm 0.0$ & $0.2 \pm 0.0$ & $3.3 \pm 0.1$ & $-0.7 \pm 0.1$ & $2.6 \pm 0.1$ \\
$\mathrm{R} 425 \mathrm{~A}$ & $2.4 \pm 0.0$ & $-0.3 \pm 0.1$ & $0.2 \pm 0.2$ & $0.1 \pm 0.0$ & $2.4 \pm 0.0$ & $-0.1 \pm 0.0$ & $2.3 \pm 0.0$ \\
$\mathrm{~N} 410 \mathrm{~A}$ & $1.1 \pm 0.4$ & $1.5 \pm 0.9$ & $-0.8 \pm 0.2$ & $0.1 \pm 0.0$ & $1.8 \pm 0.3$ & $-0.4 \pm 0.2$ & $1.4 \pm 0.2$ \\
$\mathrm{~L} 172 \mathrm{~A}$ & $1.1 \pm 0.3$ & $0.1 \pm 0.0$ & $-0.1 \pm 0.1$ & $0.1 \pm 0.0$ & $1.2 \pm 0.4$ & $-0.1 \pm 0.0$ & $1.1 \pm 0.3$ \\
$\mathrm{H} 171 \mathrm{~A}$ & $0.9 \pm 0.0$ & $0.3 \pm 0.1$ & $-0.2 \pm 0.0$ & $0.0 \pm 0.0$ & $1.0 \pm 0.1$ & $-0.0 \pm 0.0$ & $1.0 \pm 0.1$ \\
$\mathrm{Y} 176 \mathrm{~A}$ & $1.3 \pm 0.1$ & $-0.1 \pm 0.1$ & $-0.3 \pm 0.1$ & $0.2 \pm 0.0$ & $1.1 \pm 0.1$ & $-0.3 \pm 0.1$ & $0.8 \pm 0.2$ \\
$\mathrm{E} 98$ 'A & $1.0 \pm 0.1$ & $-0.0 \pm 0.0$ & $-0.3 \pm 0.1$ & $0.1 \pm 0.0$ & $0.8 \pm 0.1$ & $-0.0 \pm 0.0$ & $0.7 \pm 0.1$ \\
S226A & $0.5 \pm 0.1$ & $0.1 \pm 0.1$ & $-0.1 \pm 0.1$ & $0.0 \pm 0.0$ & $0.5 \pm 0.1$ & $-0.1 \pm 0.1$ & $0.4 \pm 0.0$ \\
I183A & $0.3 \pm 0.1$ & $0.0 \pm 0.0$ & $-0.1 \pm 0.0$ & $0.0 \pm 0.0$ & $0.3 \pm 0.1$ & $-0.0 \pm 0.0$ & $0.3 \pm 0.1$ \\
E397A & $0.2 \pm 0.1$ & $-0.9 \pm 0.4$ & $1.0 \pm 0.4$ & $0.0 \pm 0.0$ & $0.3 \pm 0.1$ & $-0.0 \pm 0.0$ & $0.3 \pm 0.1$ \\
T411A & $0.3 \pm 0.2$ & $0.0 \pm 1.0$ & $0.0 \pm 0.5$ & $0.0 \pm 0.0$ & $0.3 \pm 0.2$ & $-0.1 \pm 0.2$ & $0.2 \pm 0.1$ \\
I74A & $0.1 \pm 0.0$ & $0.0 \pm 0.0$ & $0.1 \pm 0.0$ & $0.0 \pm 0.0$ & $0.2 \pm 0.0$ & $0.0 \pm 0.0$ & $0.2 \pm 0.0$ \\
\hline Total & $17.4 \pm 0.4$ & $1.2 \pm 3.2$ & $-2.4 \pm 1.8$ & $1.3 \pm 0.1$ & $17.5 \pm 1.8$ & $-2.3 \pm 1.0$ & $15.2 \pm 0.9$ \\
\hline
\end{tabular}


Table S13. Same as Table S1 but for compound 12.

\begin{tabular}{cccccccc}
\hline Residue & $\Delta \Delta \mathrm{E}_{\mathrm{vdw}}$ & $\Delta \Delta \mathrm{E}_{\text {ele }}$ & $\Delta \Delta \mathrm{GB}$ & $\Delta \Delta \mathrm{NP}$ & $\Delta \Delta \mathrm{H}$ & IE & $\Delta \Delta G_{\text {bind }}^{x \rightarrow a}$ \\
\hline Y105'A & $5.0 \pm 0.3$ & $-0.2 \pm 0.2$ & $-0.7 \pm 0.1$ & $0.5 \pm 0.0$ & $4.6 \pm 0.2$ & $-1.4 \pm 0.1$ & $3.1 \pm 0.3$ \\
$\mathrm{~L} 172 \mathrm{~A}$ & $2.0 \pm 0.0$ & $0.1 \pm 0.0$ & $-0.3 \pm 0.0$ & $0.1 \pm 0.0$ & $1.9 \pm 0.0$ & $-0.2 \pm 0.0$ & $1.6 \pm 0.0$ \\
$\mathrm{R} 425 \mathrm{~A}$ & $1.4 \pm 0.1$ & $-0.1 \pm 0.1$ & $0.1 \pm 0.0$ & $0.1 \pm 0.0$ & $1.5 \pm 0.1$ & $-0.1 \pm 0.0$ & $1.5 \pm 0.1$ \\
$\mathrm{H} 171 \mathrm{~A}$ & $1.3 \pm 0.0$ & $0.2 \pm 0.0$ & $-0.2 \pm 0.0$ & $0.0 \pm 0.0$ & $1.3 \pm 0.0$ & $0.0 \pm 0.0$ & $1.3 \pm 0.1$ \\
$\mathrm{~K} 409 \mathrm{~A}$ & $1.5 \pm 0.0$ & $0.3 \pm 0.1$ & $-0.6 \pm 0.1$ & $0.2 \pm 0.0$ & $1.5 \pm 0.1$ & $-0.4 \pm 0.2$ & $1.1 \pm 0.2$ \\
$\mathrm{E} 98^{\prime} \mathrm{A}$ & $1.4 \pm 0.1$ & $0.2 \pm 0.0$ & $-0.8 \pm 0.1$ & $0.2 \pm 0.0$ & $1.0 \pm 0.1$ & $-0.1 \pm 0.0$ & $0.9 \pm 0.1$ \\
$\mathrm{Y} 176 \mathrm{~A}$ & $1.1 \pm 0.1$ & $0.1 \pm 0.0$ & $-0.3 \pm 0.0$ & $0.1 \pm 0.0$ & $0.9 \pm 0.0$ & $0.0 \pm 0.0$ & $0.9 \pm 0.0$ \\
F320'A & $0.9 \pm 0.0$ & $-0.1 \pm 0.0$ & $-0.1 \pm 0.0$ & $0.0 \pm 0.0$ & $0.7 \pm 0.0$ & $0.0 \pm 0.0$ & $0.7 \pm 0.0$ \\
N410A & $0.8 \pm 0.0$ & $0.2 \pm 0.0$ & $-0.3 \pm 0.0$ & $0.1 \pm 0.0$ & $0.7 \pm 0.0$ & $-0.1 \pm 0.0$ & $0.7 \pm 0.0$ \\
S226A & $0.5 \pm 0.0$ & $0.4 \pm 0.1$ & $-0.1 \pm 0.0$ & $0.0 \pm 0.0$ & $0.7 \pm 0.1$ & $-0.1 \pm 0.0$ & $0.6 \pm 0.0$ \\
N408A & $0.7 \pm 0.1$ & $0.1 \pm 0.0$ & $-0.2 \pm 0.0$ & $0.1 \pm 0.0$ & $0.7 \pm 0.0$ & $-0.1 \pm 0.0$ & $0.6 \pm 0.0$ \\
Y106'A & $1.0 \pm 0.1$ & $0.0 \pm 0.0$ & $0.0 \pm 0.0$ & $0.1 \pm 0.0$ & $1.1 \pm 0.1$ & $-0.8 \pm 0.1$ & $0.3 \pm 0.1$ \\
I183A & $0.3 \pm 0.0$ & $0.1 \pm 0.0$ & $-0.2 \pm 0.0$ & $0.0 \pm 0.0$ & $0.3 \pm 0.0$ & $0.0 \pm 0.0$ & $0.3 \pm 0.0$ \\
I74A & $0.1 \pm 0.0$ & $0.0 \pm 0.0$ & $0.1 \pm 0.0$ & $0.0 \pm 0.0$ & $0.2 \pm 0.0$ & $0.0 \pm 0.0$ & $0.2 \pm 0.0$ \\
\hline Total & $17.9 \pm 0.3$ & $1.4 \pm 0.3$ & $-3.8 \pm 0.2$ & $1.6 \pm 0.0$ & $17.0 \pm 0.1$ & $-3.3 \pm 0.3$ & $13.8 \pm 0.3$ \\
\hline
\end{tabular}


Table S14. Same as Table S1 but for compound 13.

\begin{tabular}{cccccccc}
\hline Residue & $\Delta \Delta \mathrm{E}_{\mathrm{vdw}}$ & $\Delta \Delta \mathrm{E}_{\text {ele }}$ & $\Delta \Delta \mathrm{GB}$ & $\Delta \Delta \mathrm{NP}$ & $\Delta \Delta \mathrm{H}$ & $\mathrm{IE}$ & $\Delta \Delta G_{\text {bind }}^{x \rightarrow a}$ \\
\hline Y105'A & $4.1 \pm 0.2$ & $-0.1 \pm 0.0$ & $-0.4 \pm 0.0$ & $0.3 \pm 0.0$ & $3.9 \pm 0.1$ & $-0.6 \pm 0.1$ & $3.3 \pm 0.2$ \\
$\mathrm{R} 425 \mathrm{~A}$ & $1.7 \pm 0.1$ & $-0.3 \pm 0.0$ & $0.3 \pm 0.0$ & $0.1 \pm 0.0$ & $1.8 \pm 0.1$ & $0.0 \pm 0.0$ & $1.7 \pm 0.1$ \\
$\mathrm{~K} 409 \mathrm{~A}$ & $1.4 \pm 0.1$ & $1.9 \pm 0.0$ & $-1.8 \pm 0.0$ & $0.3 \pm 0.0$ & $1.6 \pm 0.1$ & $-0.1 \pm 0.0$ & $1.6 \pm 0.1$ \\
$\mathrm{~L} 172 \mathrm{~A}$ & $1.4 \pm 0.1$ & $0.1 \pm 0.0$ & $-0.1 \pm 0.0$ & $0.1 \pm 0.0$ & $1.5 \pm 0.1$ & $-0.2 \pm 0.1$ & $1.3 \pm 0.1$ \\
$\mathrm{H} 171 \mathrm{~A}$ & $1.2 \pm 0.1$ & $-0.1 \pm 0.0$ & $0.1 \pm 0.0$ & $0.0 \pm 0.0$ & $1.2 \pm 0.1$ & $0.0 \pm 0.1$ & $1.2 \pm 0.0$ \\
$\mathrm{E} 98^{\prime} \mathrm{A}$ & $1.5 \pm 0.1$ & $0.5 \pm 0.1$ & $-1.0 \pm 0.1$ & $0.2 \pm 0.0$ & $1.2 \pm 0.1$ & $-0.1 \pm 0.0$ & $1.1 \pm 0.1$ \\
$\mathrm{~N} 410 \mathrm{~A}$ & $1.1 \pm 0.1$ & $0.3 \pm 0.1$ & $-0.4 \pm 0.1$ & $0.1 \pm 0.0$ & $1.1 \pm 0.2$ & $-0.2 \pm 0.1$ & $0.9 \pm 0.1$ \\
$\mathrm{~S} 226 \mathrm{~A}$ & $0.5 \pm 0.0$ & $0.5 \pm 0.2$ & $-0.2 \pm 0.0$ & $0.0 \pm 0.0$ & $0.8 \pm 0.2$ & $-0.1 \pm 0.0$ & $0.7 \pm 0.2$ \\
$\mathrm{~N} 408 \mathrm{~A}$ & $1.0 \pm 0.1$ & $0.1 \pm 0.0$ & $-0.3 \pm 0.0$ & $0.1 \pm 0.0$ & $0.8 \pm 0.2$ & $-0.1 \pm 0.1$ & $0.7 \pm 0.2$ \\
L166A & $1.9 \pm 0.2$ & $0.0 \pm 0.0$ & $-0.1 \pm 0.0$ & $0.2 \pm 0.0$ & $1.9 \pm 0.2$ & $-1.2 \pm 0.3$ & $0.7 \pm 0.5$ \\
F320'A & $0.7 \pm 0.1$ & $-0.1 \pm 0.0$ & $0.0 \pm 0.0$ & $0.0 \pm 0.0$ & $0.5 \pm 0.1$ & $0.0 \pm 0.0$ & $0.5 \pm 0.1$ \\
I74A & $0.1 \pm 0.0$ & $0.0 \pm 0.0$ & $0.1 \pm 0.0$ & $0.0 \pm 0.0$ & $0.2 \pm 0.0$ & $0.0 \pm 0.0$ & $0.2 \pm 0.0$ \\
H254A & $0.2 \pm 0.0$ & $-0.1 \pm 0.0$ & $0.1 \pm 0.0$ & $0.0 \pm 0.0$ & $0.2 \pm 0.0$ & $0.0 \pm 0.0$ & $0.2 \pm 0.0$ \\
E397A & $0.1 \pm 0.0$ & $-0.8 \pm 0.0$ & $0.8 \pm 0.0$ & $0.0 \pm 0.0$ & $0.2 \pm 0.0$ & $0.0 \pm 0.0$ & $0.2 \pm 0.0$ \\
\hline Total & $16.8 \pm 0.1$ & $1.7 \pm 0.8$ & $-3.0 \pm 0.6$ & $1.4 \pm 0.0$ & $16.9 \pm 0.3$ & $-2.5 \pm 0.1$ & $14.3 \pm 0.3$ \\
\hline
\end{tabular}


Table S15. Same as Table S1 but for compound 14.

\begin{tabular}{|c|c|c|c|c|c|c|c|}
\hline Residue & $\Delta \Delta \mathrm{E}_{\mathrm{vdw}}$ & $\Delta \Delta \mathrm{E}_{\text {ele }}$ & $\Delta \Delta \mathrm{GB}$ & $\Delta \Delta \mathrm{NP}$ & $\Delta \Delta \mathrm{H}$ & IE & $\Delta \Delta G_{\text {bind }}^{x \rightarrow a}$ \\
\hline Y105'A & $4.4 \pm 0.1$ & $-0.1 \pm 0.0$ & $-0.4 \pm 0.0$ & $0.3 \pm 0.0$ & $4.3 \pm 0.1$ & $-0.6 \pm 0.0$ & $3.7 \pm 0.1$ \\
\hline R425A & $1.7 \pm 0.1$ & $-0.3 \pm 0.0$ & $0.3 \pm 0.0$ & $0.1 \pm 0.0$ & $1.8 \pm 0.0$ & $0.0 \pm 0.0$ & $1.7 \pm 0.0$ \\
\hline K409A & $1.4 \pm 0.1$ & $1.9 \pm 0.0$ & $-1.9 \pm 0.0$ & $0.3 \pm 0.0$ & $1.7 \pm 0.1$ & $-0.1 \pm 0.1$ & $1.6 \pm 0.1$ \\
\hline L172A & $1.7 \pm 0.0$ & $0.1 \pm 0.0$ & $-0.1 \pm 0.0$ & $0.1 \pm 0.0$ & $1.7 \pm 0.0$ & $-0.1 \pm 0.0$ & $1.6 \pm 0.1$ \\
\hline H171A & $1.3 \pm 0.0$ & $-0.1 \pm 0.0$ & $0.1 \pm 0.0$ & $0.0 \pm 0.0$ & $1.3 \pm 0.0$ & $0.0 \pm 0.0$ & $1.3 \pm 0.0$ \\
\hline E98'A & $1.5 \pm 0.1$ & $0.4 \pm 0.0$ & $-0.9 \pm 0.0$ & $0.2 \pm 0.0$ & $1.2 \pm 0.0$ & $-0.1 \pm 0.1$ & $1.1 \pm 0.1$ \\
\hline L166A & $2.0 \pm 0.1$ & $0.0 \pm 0.0$ & $-0.2 \pm 0.0$ & $0.2 \pm 0.0$ & $2.0 \pm 0.1$ & $-1.0 \pm 0.1$ & $1.0 \pm 0.2$ \\
\hline N410A & $1.1 \pm 0.1$ & $0.4 \pm 0.1$ & $-0.5 \pm 0.0$ & $0.1 \pm 0.0$ & $1.2 \pm 0.1$ & $-0.1 \pm 0.0$ & $1.0 \pm 0.1$ \\
\hline N408A & $1.0 \pm 0.1$ & $0.2 \pm 0.0$ & $-0.3 \pm 0.0$ & $0.1 \pm 0.0$ & $1.0 \pm 0.0$ & $-0.1 \pm 0.0$ & $0.9 \pm 0.1$ \\
\hline S226A & $0.5 \pm 0.0$ & $0.5 \pm 0.1$ & $-0.2 \pm 0.0$ & $0.0 \pm 0.0$ & $0.8 \pm 0.1$ & $-0.1 \pm 0.0$ & $0.7 \pm 0.1$ \\
\hline Y176A & $0.7 \pm 0.2$ & $0.0 \pm 0.0$ & $0.0 \pm 0.0$ & $0.0 \pm 0.0$ & $0.6 \pm 0.0$ & $0.0 \pm 0.0$ & $0.6 \pm 0.0$ \\
\hline F320'A & $0.7 \pm 0.0$ & $-0.1 \pm 0.0$ & $0.0 \pm 0.0$ & $0.0 \pm 0.0$ & $0.6 \pm 0.0$ & $0.0 \pm 0.0$ & $0.6 \pm 0.0$ \\
\hline $\mathrm{I} 74 \mathrm{~A}$ & $0.1 \pm 0.0$ & $0.0 \pm 0.0$ & $0.1 \pm 0.0$ & $0.0 \pm 0.0$ & $0.2 \pm 0.0$ & $0.0 \pm 0.0$ & $0.2 \pm 0.0$ \\
\hline I183A & $0.2 \pm 0.0$ & $0.0 \pm 0.0$ & $0.0 \pm 0.0$ & $0.0 \pm 0.0$ & $0.2 \pm 0.0$ & $0.0 \pm 0.0$ & $0.2 \pm 0.0$ \\
\hline E397A & $0.2 \pm 0.0$ & $-0.8 \pm 0.0$ & $0.9 \pm 0.0$ & $0.0 \pm 0.0$ & $0.2 \pm 0.0$ & $0.0 \pm 0.0$ & $0.2 \pm 0.0$ \\
\hline $\mathrm{H} 254 \mathrm{~A}$ & $0.2 \pm 0.0$ & $0.0 \pm 0.0$ & $0.0 \pm 0.0$ & $0.0 \pm 0.0$ & $0.2 \pm 0.0$ & $0.0 \pm 0.0$ & $0.2 \pm 0.0$ \\
\hline Total & $18.5 \pm 0.2$ & $1.9 \pm 0.1$ & $-3.0 \pm 0.2$ & $1.4 \pm 0.1$ & $18.7 \pm 0.3$ & $-2.1 \pm 0.2$ & $16.5 \pm 0.1$ \\
\hline
\end{tabular}


Table S16. Same as Table S1 but for compound 15.

\begin{tabular}{|c|c|c|c|c|c|c|c|}
\hline Residue & $\Delta \Delta \mathrm{E}_{\mathrm{vdw}}$ & $\Delta \Delta \mathrm{E}_{\text {ele }}$ & $\Delta \Delta \mathrm{GB}$ & $\Delta \Delta \mathrm{NP}$ & $\Delta \Delta \mathrm{H}$ & IE & $\Delta \Delta G_{\text {bind }}^{x \rightarrow a}$ \\
\hline Y105'A & $5.3 \pm 0.2$ & $0.2 \pm 0.2$ & $-0.8 \pm 0.1$ & $0.5 \pm 0.0$ & $5.2 \pm 0.1$ & $-0.4 \pm 0.1$ & $4.8 \pm 0.1$ \\
\hline L166A & $3.3 \pm 0.1$ & $0.1 \pm 0.0$ & $-0.2 \pm 0.0$ & $0.2 \pm 0.0$ & $3.4 \pm 0.1$ & $-0.4 \pm 0.0$ & $2.9 \pm 0.1$ \\
\hline $\mathrm{R} 425 \mathrm{~A}$ & $2.2 \pm 0.2$ & $-0.4 \pm 0.1$ & $0.3 \pm 0.1$ & $0.1 \pm 0.0$ & $2.2 \pm 0.1$ & $-0.1 \pm 0.0$ & $2.1 \pm 0.2$ \\
\hline L172A & $1.8 \pm 0.1$ & $0.0 \pm 0.0$ & $-0.1 \pm 0.0$ & $0.1 \pm 0.0$ & $1.8 \pm 0.1$ & $-0.2 \pm 0.2$ & $1.7 \pm 0.0$ \\
\hline Y176A & $1.4 \pm 0.1$ & $-0.1 \pm 0.0$ & $-0.1 \pm 0.0$ & $0.1 \pm 0.0$ & $1.3 \pm 0.1$ & $-0.1 \pm 0.0$ & $1.2 \pm 0.1$ \\
\hline N410A & $1.4 \pm 0.2$ & $0.7 \pm 0.2$ & $-0.7 \pm 0.1$ & $0.1 \pm 0.0$ & $1.5 \pm 0.2$ & $-0.3 \pm 0.1$ & $1.2 \pm 0.2$ \\
\hline H171A & $1.0 \pm 0.1$ & $-0.1 \pm 0.0$ & $0.1 \pm 0.0$ & $0.0 \pm 0.0$ & $1.1 \pm 0.1$ & $0.0 \pm 0.1$ & $1.1 \pm 0.2$ \\
\hline E98'A & $1.1 \pm 0.1$ & $0.3 \pm 0.1$ & $-0.6 \pm 0.1$ & $0.1 \pm 0.0$ & $0.9 \pm 0.1$ & $-0.1 \pm 0.0$ & $0.9 \pm 0.1$ \\
\hline K409A & $0.8 \pm 0.3$ & $2.3 \pm 0.1$ & $-2.3 \pm 0.1$ & $0.3 \pm 0.0$ & $1.1 \pm 0.3$ & $-0.3 \pm 0.2$ & $0.8 \pm 0.5$ \\
\hline I183A & $0.9 \pm 0.0$ & $-0.1 \pm 0.0$ & $-0.1 \pm 0.0$ & $0.1 \pm 0.0$ & $0.9 \pm 0.0$ & $-0.1 \pm 0.1$ & $0.8 \pm 0.0$ \\
\hline N408A & $1.0 \pm 0.0$ & $0.1 \pm 0.0$ & $-0.4 \pm 0.0$ & $0.1 \pm 0.0$ & $0.8 \pm 0.1$ & $-0.1 \pm 0.1$ & $0.6 \pm 0.1$ \\
\hline S226A & $0.5 \pm 0.0$ & $0.2 \pm 0.1$ & $-0.1 \pm 0.0$ & $0.0 \pm 0.0$ & $0.6 \pm 0.1$ & $-0.1 \pm 0.0$ & $0.5 \pm 0.1$ \\
\hline F320'A & $0.6 \pm 0.1$ & $0.0 \pm 0.0$ & $-0.2 \pm 0.0$ & $0.0 \pm 0.0$ & $0.4 \pm 0.1$ & $0.0 \pm 0.0$ & $0.4 \pm 0.1$ \\
\hline E397A & $0.2 \pm 0.0$ & $-1.1 \pm 0.1$ & $1.2 \pm 0.1$ & $0.0 \pm 0.0$ & $0.3 \pm 0.0$ & $0.0 \pm 0.0$ & $0.3 \pm 0.0$ \\
\hline $\mathrm{I} 74 \mathrm{~A}$ & $0.1 \pm 0.0$ & $0.0 \pm 0.0$ & $0.1 \pm 0.0$ & $0.0 \pm 0.0$ & $0.2 \pm 0.0$ & $0.0 \pm 0.0$ & $0.2 \pm 0.0$ \\
\hline $\mathrm{T} 411 \mathrm{~A}$ & $0.4 \pm 0.1$ & $-0.5 \pm 0.0$ & $0.3 \pm 0.0$ & $0.0 \pm 0.0$ & $0.2 \pm 0.0$ & $0.0 \pm 0.1$ & $0.2 \pm 0.0$ \\
\hline F317'A & $0.4 \pm 0.0$ & $0.1 \pm 0.1$ & $-0.3 \pm 0.1$ & $0.0 \pm 0.0$ & $0.2 \pm 0.1$ & $-0.1 \pm 0.0$ & $0.2 \pm 0.1$ \\
\hline $\mathrm{H} 254 \mathrm{~A}$ & $0.2 \pm 0.0$ & $0.0 \pm 0.0$ & $0.0 \pm 0.0$ & $0.0 \pm 0.0$ & $0.2 \pm 0.0$ & $0.0 \pm 0.0$ & $0.2 \pm 0.0$ \\
\hline Total & $22.6 \pm 0.6$ & $1.8 \pm 0.5$ & $-4.0 \pm 0.3$ & $1.8 \pm 0.0$ & $22.4 \pm 0.4$ & $-2.2 \pm 0.1$ & $20.2 \pm 0.2$ \\
\hline
\end{tabular}


Table S17. Same as Table S1 but for compound 16.

\begin{tabular}{|c|c|c|c|c|c|c|c|}
\hline Residue & $\Delta \Delta \mathrm{Evdw}$ & $\Delta \Delta$ Eele & $\Delta \Delta \mathrm{GB}$ & $\Delta \Delta \mathrm{NP}$ & $\Delta \Delta \mathrm{H}$ & IE & $\Delta \Delta G_{b i n d}^{x \rightarrow a}$ \\
\hline Y105'A & $4.4 \pm 0.1$ & $-0.0 \pm 0.1$ & $-0.7 \pm 0.1$ & $0.4 \pm 0.0$ & $4.1 \pm 0.3$ & $-0.6 \pm 0.3$ & $3.5 \pm 0.5$ \\
\hline $\mathrm{R} 425 \mathrm{~A}$ & $2.0 \pm 0.3$ & $-0.1 \pm 0.1$ & $0.0 \pm 0.1$ & $0.1 \pm 0.0$ & $2.0 \pm 0.3$ & $-0.1 \pm 0.1$ & $1.9 \pm 0.2$ \\
\hline L172A & $1.6 \pm 0.4$ & $-0.0 \pm 0.0$ & $0.0 \pm 0.0$ & $0.1 \pm 0.0$ & $1.7 \pm 0.4$ & $-0.2 \pm 0.1$ & $1.5 \pm 0.3$ \\
\hline N410A & $1.3 \pm 0.2$ & $0.9 \pm 0.3$ & $-0.7 \pm 0.2$ & $0.1 \pm 0.0$ & $1.6 \pm 0.3$ & $-0.3 \pm 0.1$ & $1.3 \pm 0.2$ \\
\hline Y176A & $1.6 \pm 0.1$ & $0.0 \pm 0.0$ & $-0.3 \pm 0.0$ & $0.2 \pm 0.0$ & $1.5 \pm 0.1$ & $-0.2 \pm 0.0$ & $1.3 \pm 0.1$ \\
\hline L166A & $2.8 \pm 0.6$ & $0.1 \pm 0.0$ & $-0.4 \pm 0.0$ & $0.2 \pm 0.0$ & $2.8 \pm 0.6$ & $-1.4 \pm 0.4$ & $1.5 \pm 0.6$ \\
\hline H171A & $1.0 \pm 0.2$ & $0.1 \pm 0.1$ & $-0.1 \pm 0.0$ & $0.0 \pm 0.0$ & $1.1 \pm 0.2$ & $-0.0 \pm 0.0$ & $1.1 \pm 0.2$ \\
\hline K409A & $0.9 \pm 0.4$ & $2.6 \pm 0.1$ & $-2.5 \pm 0.1$ & $0.3 \pm 0.0$ & $1.3 \pm 0.4$ & $-0.3 \pm 0.2$ & $1.0 \pm 0.6$ \\
\hline E98'A & $1.1 \pm 0.3$ & $-0.1 \pm 0.1$ & $-0.3 \pm 0.2$ & $0.2 \pm 0.0$ & $0.9 \pm 0.3$ & $-0.1 \pm 0.0$ & $0.8 \pm 0.3$ \\
\hline I183A & $0.6 \pm 0.2$ & $-0.1 \pm 0.0$ & $0.1 \pm 0.0$ & $0.1 \pm 0.0$ & $0.7 \pm 0.3$ & $-0.0 \pm 0.0$ & $0.6 \pm 0.3$ \\
\hline N408A & $0.9 \pm 0.2$ & $0.2 \pm 0.1$ & $-0.4 \pm 0.0$ & $0.1 \pm 0.0$ & $0.8 \pm 0.3$ & $-0.2 \pm 0.1$ & $0.6 \pm 0.4$ \\
\hline S226A & $0.4 \pm 0.1$ & $0.2 \pm 0.2$ & $-0.1 \pm 0.0$ & $0.0 \pm 0.0$ & $0.6 \pm 0.2$ & $-0.1 \pm 0.0$ & $0.5 \pm 0.2$ \\
\hline E397A & $0.2 \pm 0.0$ & $-1.3 \pm 0.1$ & $1.3 \pm 0.1$ & $0.0 \pm 0.0$ & $0.3 \pm 0.1$ & $-0.0 \pm 0.0$ & $0.2 \pm 0.1$ \\
\hline F320'A & $0.6 \pm 0.2$ & $0.0 \pm 0.0$ & $-0.3 \pm 0.0$ & $0.0 \pm 0.0$ & $0.3 \pm 0.2$ & $-0.0 \pm 0.0$ & $0.3 \pm 0.2$ \\
\hline $\mathrm{I} 74 \mathrm{~A}$ & $0.1 \pm 0.0$ & $0.0 \pm 0.0$ & $0.1 \pm 0.0$ & $0.0 \pm 0.0$ & $0.2 \pm 0.0$ & $0.0 \pm 0.0$ & $0.2 \pm 0.0$ \\
\hline Total & $19.7 \pm 0.6$ & $2.4 \pm 0.4$ & $-4.0 \pm 0.3$ & $1.6 \pm 0.0$ & $19.7 \pm 0.8$ & $-3.3 \pm 0.5$ & $16.4 \pm 0.9$ \\
\hline
\end{tabular}


Table S18. Same as Table S1 but for compound 17.

\begin{tabular}{|c|c|c|c|c|c|c|c|}
\hline Residue & $\Delta \Delta \mathrm{E}_{\mathrm{vdw}}$ & $\Delta \Delta \mathrm{E}_{\text {ele }}$ & $\Delta \Delta \mathrm{GB}$ & $\Delta \Delta \mathrm{NP}$ & $\Delta \Delta \mathrm{H}$ & IE & $\Delta \Delta G_{\text {bind }}^{x \rightarrow a}$ \\
\hline Y105'A & $3.0 \pm 0.1$ & $-0.2 \pm 0.0$ & $-0.2 \pm 0.0$ & $0.3 \pm 0.0$ & $3.0 \pm 0.1$ & $-0.3 \pm 0.0$ & $2.8 \pm 0.1$ \\
\hline N410A & $1.6 \pm 0.1$ & $1.7 \pm 0.2$ & $-1.3 \pm 0.1$ & $0.1 \pm 0.0$ & $2.1 \pm 0.0$ & $-0.2 \pm 0.0$ & $2.0 \pm 0.0$ \\
\hline $\mathrm{R} 425 \mathrm{~A}$ & $1.7 \pm 0.0$ & $-0.8 \pm 0.0$ & $0.7 \pm 0.0$ & $0.1 \pm 0.0$ & $1.7 \pm 0.0$ & $-0.0 \pm 0.0$ & $1.7 \pm 0.0$ \\
\hline K409A & $1.2 \pm 0.0$ & $0.8 \pm 0.0$ & $-0.8 \pm 0.0$ & $0.1 \pm 0.0$ & $1.3 \pm 0.0$ & $-0.2 \pm 0.0$ & $1.1 \pm 0.0$ \\
\hline L172A & $0.7 \pm 0.0$ & $0.1 \pm 0.0$ & $-0.1 \pm 0.0$ & $0.1 \pm 0.0$ & $0.7 \pm 0.0$ & $-0.0 \pm 0.0$ & $0.7 \pm 0.0$ \\
\hline H171A & $0.5 \pm 0.0$ & $-0.1 \pm 0.0$ & $0.2 \pm 0.0$ & $0.0 \pm 0.0$ & $0.5 \pm 0.0$ & $-0.0 \pm 0.0$ & $0.5 \pm 0.0$ \\
\hline Y106'A & $0.8 \pm 0.1$ & $0.0 \pm 0.0$ & $-0.0 \pm 0.0$ & $0.1 \pm 0.0$ & $0.9 \pm 0.1$ & $-0.4 \pm 0.1$ & $0.5 \pm 0.2$ \\
\hline E98'A & $0.5 \pm 0.0$ & $0.2 \pm 0.0$ & $-0.4 \pm 0.0$ & $0.1 \pm 0.0$ & $0.3 \pm 0.0$ & $-0.0 \pm 0.0$ & $0.3 \pm 0.0$ \\
\hline L166A & $1.9 \pm 0.1$ & $0.0 \pm 0.0$ & $-0.5 \pm 0.0$ & $0.2 \pm 0.0$ & $1.6 \pm 0.2$ & $-1.4 \pm 0.1$ & $0.2 \pm 0.1$ \\
\hline Total & $11.8 \pm 0.3$ & $1.8 \pm 0.2$ & $-2.4 \pm 0.1$ & $1.0 \pm 0.1$ & $12.3 \pm 0.2$ & $-2.5 \pm 0.2$ & $9.8 \pm 0.1$ \\
\hline
\end{tabular}


Table S19. Same as Table S1 but for compound 18.

\begin{tabular}{|c|c|c|c|c|c|c|c|}
\hline Residue & $\Delta \Delta \mathrm{E}_{\mathrm{vdw}}$ & $\Delta \Delta \mathrm{E}_{\text {ele }}$ & $\Delta \Delta \mathrm{GB}$ & $\Delta \Delta \mathrm{NP}$ & $\Delta \Delta \mathrm{H}$ & IE & $\Delta \Delta G_{\text {bind }}^{x \rightarrow a}$ \\
\hline Y105'A & $3.7 \pm 0.1$ & $-0.1 \pm 0.1$ & $-0.2 \pm 0.0$ & $0.3 \pm 0.0$ & $3.7 \pm 0.1$ & $-0.4 \pm 0.4$ & $3.3 \pm 0.4$ \\
\hline $\mathrm{R} 425 \mathrm{~A}$ & $2.2 \pm 0.3$ & $-0.5 \pm 0.2$ & $0.4 \pm 0.1$ & $0.1 \pm 0.0$ & $2.2 \pm 0.3$ & $-0.1 \pm 0.0$ & $2.1 \pm 0.3$ \\
\hline L166A & $2.1 \pm 0.4$ & $0.0 \pm 0.0$ & $-0.2 \pm 0.0$ & $0.2 \pm 0.0$ & $2.2 \pm 0.5$ & $-0.8 \pm 0.5$ & $1.3 \pm 1.0$ \\
\hline N410A & $1.2 \pm 0.1$ & $1.0 \pm 0.2$ & $-0.8 \pm 0.2$ & $0.1 \pm 0.0$ & $1.5 \pm 0.2$ & $-0.3 \pm 0.0$ & $1.1 \pm 0.2$ \\
\hline K409A & $1.0 \pm 0.1$ & $1.2 \pm 0.2$ & $-1.2 \pm 0.2$ & $0.1 \pm 0.0$ & $1.2 \pm 0.1$ & $-0.1 \pm 0.0$ & $1.1 \pm 0.2$ \\
\hline H171A & $1.0 \pm 0.2$ & $0.0 \pm 0.0$ & $0.0 \pm 0.0$ & $0.0 \pm 0.0$ & $1.0 \pm 0.2$ & $0.0 \pm 0.0$ & $1.0 \pm 0.2$ \\
\hline L172A & $1.0 \pm 0.2$ & $0.1 \pm 0.0$ & $-0.1 \pm 0.0$ & $0.1 \pm 0.0$ & $1.1 \pm 0.2$ & $-0.1 \pm 0.1$ & $1.0 \pm 0.1$ \\
\hline E98'A & $1.1 \pm 0.3$ & $0.2 \pm 0.1$ & $-0.5 \pm 0.2$ & $0.1 \pm 0.0$ & $0.9 \pm 0.2$ & $-0.1 \pm 0.0$ & $0.8 \pm 0.2$ \\
\hline N408A & $0.9 \pm 0.1$ & $0.0 \pm 0.1$ & $-0.3 \pm 0.0$ & $0.0 \pm 0.0$ & $0.6 \pm 0.2$ & $-0.2 \pm 0.1$ & $0.5 \pm 0.3$ \\
\hline S226A & $0.5 \pm 0.0$ & $0.2 \pm 0.2$ & $-0.1 \pm 0.0$ & $0.0 \pm 0.0$ & $0.5 \pm 0.2$ & $-0.1 \pm 0.0$ & $0.5 \pm 0.2$ \\
\hline F320'A & $0.4 \pm 0.1$ & $0.0 \pm 0.0$ & $-0.1 \pm 0.0$ & $0.0 \pm 0.0$ & $0.3 \pm 0.1$ & $0.0 \pm 0.0$ & $0.3 \pm 0.1$ \\
\hline I74A & $0.1 \pm 0.0$ & $0.0 \pm 0.0$ & $0.1 \pm 0.0$ & $0.0 \pm 0.0$ & $0.2 \pm 0.0$ & $0.0 \pm 0.0$ & $0.2 \pm 0.0$ \\
\hline T411A & $0.4 \pm 0.1$ & $-0.5 \pm 0.1$ & $0.3 \pm 0.1$ & $0.0 \pm 0.0$ & $0.2 \pm 0.1$ & $0.0 \pm 0.1$ & $0.2 \pm 0.0$ \\
\hline E397A & $0.2 \pm 0.0$ & $-0.5 \pm 0.1$ & $0.5 \pm 0.1$ & $0.0 \pm 0.0$ & $0.2 \pm 0.1$ & $0.0 \pm 0.0$ & $0.2 \pm 0.1$ \\
\hline $\mathrm{H} 254 \mathrm{~A}$ & $0.2 \pm 0.0$ & $0.0 \pm 0.0$ & $0.0 \pm 0.0$ & $0.0 \pm 0.0$ & $0.2 \pm 0.0$ & $0.0 \pm 0.0$ & $0.2 \pm 0.0$ \\
\hline Total & $15.7 \pm 0.3$ & $1.1 \pm 0.5$ & $-2.1 \pm 0.4$ & $1.1 \pm 0.0$ & $15.8 \pm 0.3$ & $-2.2 \pm 1.0$ & $13.6 \pm 0.9$ \\
\hline
\end{tabular}


Table S20. Same as Table S1 but for compound 19.

\begin{tabular}{|c|c|c|c|c|c|c|c|}
\hline Residue & $\Delta \Delta \mathrm{E}_{\mathrm{vdw}}$ & $\Delta \Delta \mathrm{E}_{\text {ele }}$ & $\Delta \Delta \mathrm{GB}$ & $\Delta \Delta \mathrm{NP}$ & $\Delta \Delta \mathrm{H}$ & IE & $\Delta \Delta G_{b i n d}^{x \rightarrow a}$ \\
\hline Y105'A & $3.0 \pm 0.1$ & $-0.2 \pm 0.0$ & $-0.2 \pm 0.0$ & $0.3 \pm 0.0$ & $2.9 \pm 0.1$ & $-0.2 \pm 0.0$ & $2.7 \pm 0.1$ \\
\hline N410A & $1.8 \pm 0.1$ & $1.6 \pm 0.1$ & $-1.3 \pm 0.0$ & $0.1 \pm 0.0$ & $2.2 \pm 0.0$ & $-0.2 \pm 0.0$ & $2.0 \pm 0.0$ \\
\hline $\mathrm{R} 425 \mathrm{~A}$ & $1.8 \pm 0.0$ & $-0.9 \pm 0.0$ & $0.8 \pm 0.0$ & $0.1 \pm 0.0$ & $1.8 \pm 0.0$ & $0.0 \pm 0.0$ & $1.7 \pm 0.0$ \\
\hline K409A & $1.2 \pm 0.1$ & $1.5 \pm 0.0$ & $-1.5 \pm 0.0$ & $0.3 \pm 0.0$ & $1.5 \pm 0.1$ & $-0.2 \pm 0.0$ & $1.3 \pm 0.1$ \\
\hline Y106'A & $0.9 \pm 0.1$ & $0.1 \pm 0.0$ & $0.0 \pm 0.0$ & $0.1 \pm 0.0$ & $1.0 \pm 0.1$ & $-0.3 \pm 0.0$ & $0.7 \pm 0.1$ \\
\hline L172A & $0.6 \pm 0.0$ & $0.1 \pm 0.0$ & $-0.1 \pm 0.0$ & $0.0 \pm 0.0$ & $0.7 \pm 0.0$ & $0.0 \pm 0.0$ & $0.6 \pm 0.0$ \\
\hline H171A & $0.5 \pm 0.0$ & $-0.3 \pm 0.0$ & $0.3 \pm 0.0$ & $0.0 \pm 0.0$ & $0.5 \pm 0.0$ & $0.0 \pm 0.0$ & $0.5 \pm 0.0$ \\
\hline L166A & $1.9 \pm 0.0$ & $0.0 \pm 0.0$ & $-0.5 \pm 0.0$ & $0.2 \pm 0.0$ & $1.6 \pm 0.1$ & $-1.2 \pm 0.1$ & $0.4 \pm 0.0$ \\
\hline E98'A & $0.5 \pm 0.0$ & $0.2 \pm 0.0$ & $-0.4 \pm 0.0$ & $0.1 \pm 0.0$ & $0.3 \pm 0.0$ & $0.0 \pm 0.0$ & $0.3 \pm 0.0$ \\
\hline $\mathrm{I} 74 \mathrm{~A}$ & $0.1 \pm 0.0$ & $0.0 \pm 0.0$ & $0.1 \pm 0.0$ & $0.0 \pm 0.0$ & $0.2 \pm 0.0$ & $0.0 \pm 0.0$ & $0.2 \pm 0.0$ \\
\hline E397A & $0.2 \pm 0.0$ & $-0.5 \pm 0.0$ & $0.5 \pm 0.0$ & $0.0 \pm 0.0$ & $0.2 \pm 0.0$ & $0.0 \pm 0.0$ & $0.2 \pm 0.0$ \\
\hline D168A & $0.0 \pm 0.0$ & $0.8 \pm 0.0$ & $-0.6 \pm 0.0$ & $0.0 \pm 0.0$ & $0.2 \pm 0.0$ & $0.0 \pm 0.0$ & $0.2 \pm 0.0$ \\
\hline Total & $12.3 \pm 0.1$ & $2.5 \pm 0.2$ & $-2.9 \pm 0.1$ & $1.1 \pm 0.1$ & $13.0 \pm 0.2$ & $-2.3 \pm 0.1$ & $10.7 \pm 0.1$ \\
\hline
\end{tabular}


Table S21. Same as Table S1 but for compound 20.

\begin{tabular}{|c|c|c|c|c|c|c|c|}
\hline Residue & $\Delta \Delta \mathrm{E}_{\mathrm{vdw}}$ & $\Delta \Delta \mathrm{E}_{\text {ele }}$ & $\Delta \Delta \mathrm{GB}$ & $\Delta \Delta \mathrm{NP}$ & $\Delta \Delta \mathrm{H}$ & IE & $\Delta \Delta G_{\text {bind }}^{x \rightarrow a}$ \\
\hline Y105'A & $3.5 \pm 0.1$ & $-0.3 \pm 0.0$ & $-0.3 \pm 0.0$ & $0.3 \pm 0.0$ & $3.3 \pm 0.1$ & $-0.3 \pm 0.0$ & $3.0 \pm 0.1$ \\
\hline R425A & $1.8 \pm 0.0$ & $-0.1 \pm 0.1$ & $0.1 \pm 0.1$ & $0.1 \pm 0.0$ & $1.8 \pm 0.0$ & $0.0 \pm 0.0$ & $1.8 \pm 0.0$ \\
\hline L166A & $2.1 \pm 0.2$ & $0.0 \pm 0.0$ & $-0.2 \pm 0.0$ & $0.2 \pm 0.0$ & $2.1 \pm 0.2$ & $-0.7 \pm 0.2$ & $1.4 \pm 0.4$ \\
\hline H171A & $1.2 \pm 0.1$ & $0.1 \pm 0.0$ & $-0.1 \pm 0.0$ & $0.0 \pm 0.0$ & $1.2 \pm 0.1$ & $0.0 \pm 0.0$ & $1.3 \pm 0.1$ \\
\hline N410A & $1.1 \pm 0.1$ & $0.9 \pm 0.7$ & $-0.6 \pm 0.3$ & $0.1 \pm 0.0$ & $1.5 \pm 0.3$ & $-0.3 \pm 0.1$ & $1.2 \pm 0.2$ \\
\hline E98'A & $1.4 \pm 0.2$ & $0.1 \pm 0.0$ & $-0.5 \pm 0.1$ & $0.2 \pm 0.0$ & $1.1 \pm 0.1$ & $-0.1 \pm 0.0$ & $1.0 \pm 0.1$ \\
\hline L172A & $1.0 \pm 0.1$ & $0.1 \pm 0.0$ & $-0.1 \pm 0.0$ & $0.1 \pm 0.0$ & $1.0 \pm 0.1$ & $-0.1 \pm 0.0$ & $1.0 \pm 0.1$ \\
\hline K409A & $0.8 \pm 0.0$ & $1.2 \pm 0.0$ & $-1.2 \pm 0.0$ & $0.1 \pm 0.0$ & $1.0 \pm 0.0$ & $-0.1 \pm 0.0$ & $0.9 \pm 0.0$ \\
\hline N408A & $0.9 \pm 0.1$ & $0.2 \pm 0.0$ & $-0.3 \pm 0.0$ & $0.1 \pm 0.0$ & $0.9 \pm 0.1$ & $-0.1 \pm 0.1$ & $0.8 \pm 0.2$ \\
\hline S226A & $0.5 \pm 0.0$ & $0.4 \pm 0.2$ & $-0.1 \pm 0.0$ & $0.0 \pm 0.0$ & $0.8 \pm 0.2$ & $-0.1 \pm 0.0$ & $0.7 \pm 0.2$ \\
\hline F320'A & $0.5 \pm 0.1$ & $0.0 \pm 0.0$ & $-0.2 \pm 0.0$ & $0.0 \pm 0.0$ & $0.4 \pm 0.0$ & $0.0 \pm 0.0$ & $0.4 \pm 0.0$ \\
\hline I74A & $0.1 \pm 0.0$ & $0.0 \pm 0.0$ & $0.1 \pm 0.0$ & $0.0 \pm 0.0$ & $0.2 \pm 0.0$ & $0.0 \pm 0.0$ & $0.2 \pm 0.0$ \\
\hline $\mathrm{H} 254 \mathrm{~A}$ & $0.2 \pm 0.0$ & $0.0 \pm 0.0$ & $0.0 \pm 0.0$ & $0.0 \pm 0.0$ & $0.2 \pm 0.0$ & $0.0 \pm 0.0$ & $0.2 \pm 0.0$ \\
\hline $\mathrm{T} 411 \mathrm{~A}$ & $0.2 \pm 0.0$ & $-0.3 \pm 0.1$ & $0.2 \pm 0.1$ & $0.0 \pm 0.0$ & $0.1 \pm 0.0$ & $0.0 \pm 0.0$ & $0.2 \pm 0.0$ \\
\hline Total & $15.3 \pm 0.6$ & $2.2 \pm 0.3$ & $-3.1 \pm 0.3$ & $1.1 \pm 0.0$ & $15.5 \pm 0.4$ & $-1.7 \pm 0.5$ & $13.8 \pm 0.9$ \\
\hline
\end{tabular}


Table S22. Same as Table S1 but for compound 21.

\begin{tabular}{|c|c|c|c|c|c|c|c|}
\hline Residue & $\Delta \Delta \mathrm{E}_{\mathrm{vdw}}$ & $\Delta \Delta \mathrm{E}_{\text {ele }}$ & $\Delta \Delta \mathrm{GB}$ & $\Delta \Delta \mathrm{NP}$ & $\Delta \Delta \mathrm{H}$ & IE & $\Delta \Delta G_{\text {bind }}^{x \rightarrow a}$ \\
\hline Y105'A & $3.7 \pm 0.5$ & $-0.1 \pm 0.1$ & $-0.3 \pm 0.1$ & $0.3 \pm 0.0$ & $3.5 \pm 0.5$ & $-0.5 \pm 0.2$ & $3.0 \pm 0.6$ \\
\hline $\mathrm{R} 425 \mathrm{~A}$ & $1.9 \pm 0.2$ & $-0.2 \pm 0.1$ & $0.2 \pm 0.1$ & $0.1 \pm 0.0$ & $2.0 \pm 0.2$ & $-0.1 \pm 0.0$ & $1.9 \pm 0.1$ \\
\hline N410A & $1.1 \pm 0.3$ & $1.2 \pm 0.7$ & $-0.8 \pm 0.3$ & $0.1 \pm 0.0$ & $1.6 \pm 0.3$ & $-0.4 \pm 0.1$ & $1.2 \pm 0.2$ \\
\hline H171A & $1.1 \pm 0.2$ & $0.2 \pm 0.0$ & $-0.2 \pm 0.0$ & $0.0 \pm 0.0$ & $1.2 \pm 0.1$ & $0.0 \pm 0.0$ & $1.2 \pm 0.2$ \\
\hline K409A & $1.0 \pm 0.2$ & $1.3 \pm 0.2$ & $-1.3 \pm 0.1$ & $0.1 \pm 0.0$ & $1.1 \pm 0.2$ & $-0.1 \pm 0.0$ & $1.0 \pm 0.2$ \\
\hline L172A & $1.1 \pm 0.2$ & $0.1 \pm 0.0$ & $-0.1 \pm 0.0$ & $0.1 \pm 0.0$ & $1.1 \pm 0.2$ & $-0.1 \pm 0.1$ & $1.0 \pm 0.1$ \\
\hline L166A & $2.4 \pm 0.4$ & $0.0 \pm 0.1$ & $-0.5 \pm 0.0$ & $0.2 \pm 0.0$ & $2.1 \pm 0.4$ & $-1.2 \pm 0.5$ & $0.9 \pm 0.7$ \\
\hline E98'A & $1.2 \pm 0.2$ & $0.0 \pm 0.1$ & $-0.4 \pm 0.2$ & $0.2 \pm 0.0$ & $1.0 \pm 0.2$ & $-0.1 \pm 0.0$ & $0.9 \pm 0.2$ \\
\hline N408A & $0.9 \pm 0.0$ & $0.2 \pm 0.1$ & $-0.3 \pm 0.0$ & $0.0 \pm 0.0$ & $0.8 \pm 0.1$ & $-0.2 \pm 0.0$ & $0.6 \pm 0.1$ \\
\hline S226A & $0.5 \pm 0.0$ & $0.3 \pm 0.1$ & $-0.1 \pm 0.0$ & $0.0 \pm 0.0$ & $0.6 \pm 0.1$ & $-0.1 \pm 0.0$ & $0.5 \pm 0.1$ \\
\hline F320'A & $0.5 \pm 0.1$ & $0.0 \pm 0.0$ & $-0.1 \pm 0.0$ & $0.0 \pm 0.0$ & $0.3 \pm 0.1$ & $0.0 \pm 0.0$ & $0.3 \pm 0.1$ \\
\hline $\mathrm{T} 411 \mathrm{~A}$ & $0.3 \pm 0.1$ & $-0.4 \pm 0.2$ & $0.2 \pm 0.1$ & $0.0 \pm 0.0$ & $0.2 \pm 0.0$ & $0.0 \pm 0.0$ & $0.2 \pm 0.1$ \\
\hline $\mathrm{I} 74 \mathrm{~A}$ & $0.1 \pm 0.0$ & $0.0 \pm 0.0$ & $0.1 \pm 0.0$ & $0.0 \pm 0.0$ & $0.2 \pm 0.0$ & $0.0 \pm 0.0$ & $0.2 \pm 0.0$ \\
\hline $\mathrm{H} 254 \mathrm{~A}$ & $0.2 \pm 0.0$ & $0.0 \pm 0.0$ & $0.0 \pm 0.0$ & $0.0 \pm 0.0$ & $0.2 \pm 0.0$ & $0.0 \pm 0.0$ & $0.2 \pm 0.0$ \\
\hline Total & $15.9 \pm 2.2$ & $2.5 \pm 0.6$ & $-3.6 \pm 0.3$ & $1.1 \pm 0.2$ & $15.9 \pm 1.7$ & $-2.8 \pm 0.6$ & $13.1 \pm 1.2$ \\
\hline
\end{tabular}


Table S23. Same as Table S1 but for compound 22.

\begin{tabular}{cccccccc}
\hline Residue & $\Delta \Delta \mathrm{E}_{\mathrm{vdw}}$ & $\Delta \Delta \mathrm{E}_{\text {ele }}$ & $\Delta \Delta \mathrm{GB}$ & $\Delta \Delta \mathrm{NP}$ & $\Delta \Delta \mathrm{H}$ & $\mathrm{IE}$ & $\Delta \Delta G_{\text {bind }}^{x \rightarrow a}$ \\
\hline Y105'A & $3.8 \pm 0.0$ & $-0.2 \pm 0.1$ & $-0.3 \pm 0.0$ & $0.3 \pm 0.0$ & $3.6 \pm 0.0$ & $-0.4 \pm 0.0$ & $3.2 \pm 0.0$ \\
$\mathrm{R} 425 \mathrm{~A}$ & $1.8 \pm 0.1$ & $-0.3 \pm 0.1$ & $0.3 \pm 0.1$ & $0.1 \pm 0.0$ & $1.8 \pm 0.1$ & $-0.1 \pm 0.0$ & $1.7 \pm 0.1$ \\
$\mathrm{~K} 409 \mathrm{~A}$ & $1.3 \pm 0.0$ & $2.2 \pm 0.1$ & $-2.1 \pm 0.1$ & $0.3 \pm 0.0$ & $1.6 \pm 0.0$ & $-0.1 \pm 0.0$ & $1.5 \pm 0.0$ \\
$\mathrm{~N} 410 \mathrm{~A}$ & $1.1 \pm 0.1$ & $1.4 \pm 0.8$ & $-0.9 \pm 0.3$ & $0.1 \pm 0.0$ & $1.7 \pm 0.4$ & $-0.3 \pm 0.1$ & $1.4 \pm 0.3$ \\
$\mathrm{H} 171 \mathrm{~A}$ & $1.2 \pm 0.2$ & $-0.1 \pm 0.0$ & $0.1 \pm 0.0$ & $0.0 \pm 0.0$ & $1.2 \pm 0.1$ & $0.0 \pm 0.0$ & $1.2 \pm 0.1$ \\
L172A & $1.0 \pm 0.0$ & $0.0 \pm 0.0$ & $0.0 \pm 0.0$ & $0.1 \pm 0.0$ & $1.1 \pm 0.0$ & $-0.1 \pm 0.0$ & $1.0 \pm 0.0$ \\
$\mathrm{E} 98^{\prime} \mathrm{A}$ & $1.2 \pm 0.2$ & $0.3 \pm 0.0$ & $-0.7 \pm 0.1$ & $0.2 \pm 0.0$ & $1.0 \pm 0.1$ & $-0.1 \pm 0.0$ & $0.9 \pm 0.1$ \\
L166A & $1.8 \pm 0.2$ & $-0.1 \pm 0.0$ & $-0.1 \pm 0.0$ & $0.2 \pm 0.0$ & $1.8 \pm 0.2$ & $-0.9 \pm 0.1$ & $0.9 \pm 0.3$ \\
N408A & $0.9 \pm 0.2$ & $0.1 \pm 0.0$ & $-0.3 \pm 0.0$ & $0.1 \pm 0.0$ & $0.8 \pm 0.2$ & $-0.2 \pm 0.1$ & $0.6 \pm 0.3$ \\
S226A & $0.5 \pm 0.0$ & $0.3 \pm 0.2$ & $-0.1 \pm 0.0$ & $0.0 \pm 0.0$ & $0.6 \pm 0.2$ & $-0.1 \pm 0.0$ & $0.5 \pm 0.2$ \\
F320'A & $0.5 \pm 0.1$ & $0.0 \pm 0.0$ & $-0.1 \pm 0.0$ & $0.0 \pm 0.0$ & $0.3 \pm 0.1$ & $0.0 \pm 0.0$ & $0.3 \pm 0.1$ \\
I74A & $0.1 \pm 0.0$ & $0.0 \pm 0.0$ & $0.1 \pm 0.0$ & $0.0 \pm 0.0$ & $0.2 \pm 0.0$ & $0.0 \pm 0.0$ & $0.2 \pm 0.0$ \\
T411A & $0.3 \pm 0.1$ & $-0.4 \pm 0.1$ & $0.3 \pm 0.1$ & $0.0 \pm 0.0$ & $0.2 \pm 0.0$ & $0.0 \pm 0.0$ & $0.2 \pm 0.0$ \\
H254A & $0.2 \pm 0.0$ & $0.0 \pm 0.0$ & $0.0 \pm 0.0$ & $0.0 \pm 0.0$ & $0.2 \pm 0.0$ & $0.0 \pm 0.0$ & $0.2 \pm 0.0$ \\
\hline Total & $15.4 \pm 0.7$ & $3.1 \pm 0.5$ & $-3.9 \pm 0.6$ & $1.3 \pm 0.0$ & $15.9 \pm 0.6$ & $-2.2 \pm 0.3$ & $13.7 \pm 0.8$ \\
\hline
\end{tabular}


Table S24. Same as Table S1 but for compound 23.

\begin{tabular}{cccccccc}
\hline Residue & $\Delta \Delta \mathrm{E}_{\mathrm{vdw}}$ & $\Delta \Delta \mathrm{E}_{\text {ele }}$ & $\Delta \Delta \mathrm{GB}$ & $\Delta \Delta \mathrm{NP}$ & $\Delta \Delta \mathrm{H}$ & $\mathrm{IE}$ & $\Delta \Delta G_{\text {bind }}^{x \rightarrow a}$ \\
\hline Y105'A & $3.1 \pm 0.0$ & $-0.2 \pm 0.0$ & $-0.2 \pm 0.0$ & $0.3 \pm 0.0$ & $3.0 \pm 0.0$ & $-0.3 \pm 0.0$ & $2.8 \pm 0.0$ \\
$\mathrm{~N} 410 \mathrm{~A}$ & $1.8 \pm 0.0$ & $1.4 \pm 0.1$ & $-1.1 \pm 0.0$ & $0.1 \pm 0.0$ & $2.1 \pm 0.0$ & $-0.2 \pm 0.0$ & $1.9 \pm 0.0$ \\
$\mathrm{R} 425 \mathrm{~A}$ & $1.8 \pm 0.0$ & $-0.4 \pm 0.0$ & $0.4 \pm 0.0$ & $0.1 \pm 0.0$ & $1.8 \pm 0.0$ & $0.0 \pm 0.0$ & $1.8 \pm 0.0$ \\
$\mathrm{~K} 409 \mathrm{~A}$ & $1.2 \pm 0.0$ & $1.3 \pm 0.0$ & $-1.3 \pm 0.0$ & $0.1 \pm 0.0$ & $1.3 \pm 0.0$ & $-0.2 \pm 0.0$ & $1.2 \pm 0.1$ \\
L166A & $2.1 \pm 0.1$ & $-0.1 \pm 0.0$ & $-0.3 \pm 0.0$ & $0.2 \pm 0.0$ & $1.9 \pm 0.1$ & $-1.0 \pm 0.3$ & $0.9 \pm 0.4$ \\
L172A & $0.6 \pm 0.0$ & $0.0 \pm 0.0$ & $0.0 \pm 0.0$ & $0.0 \pm 0.0$ & $0.6 \pm 0.0$ & $0.0 \pm 0.0$ & $0.6 \pm 0.0$ \\
H171A & $0.5 \pm 0.0$ & $0.0 \pm 0.0$ & $0.1 \pm 0.0$ & $0.0 \pm 0.0$ & $0.6 \pm 0.0$ & $0.0 \pm 0.0$ & $0.5 \pm 0.0$ \\
Y106'A & $0.8 \pm 0.1$ & $0.1 \pm 0.0$ & $0.0 \pm 0.0$ & $0.1 \pm 0.0$ & $0.9 \pm 0.1$ & $-0.4 \pm 0.0$ & $0.5 \pm 0.1$ \\
E98'A & $0.5 \pm 0.0$ & $-0.1 \pm 0.0$ & $-0.2 \pm 0.0$ & $0.1 \pm 0.0$ & $0.3 \pm 0.0$ & $0.0 \pm 0.0$ & $0.3 \pm 0.0$ \\
I74A & $0.1 \pm 0.0$ & $0.0 \pm 0.0$ & $0.1 \pm 0.0$ & $0.0 \pm 0.0$ & $0.2 \pm 0.0$ & $0.0 \pm 0.0$ & $0.2 \pm 0.0$ \\
D168A & $0.0 \pm 0.0$ & $0.7 \pm 0.0$ & $-0.6 \pm 0.0$ & $0.0 \pm 0.0$ & $0.2 \pm 0.0$ & $0.0 \pm 0.0$ & $0.2 \pm 0.0$ \\
\hline Total & $12.3 \pm 0.2$ & $2.8 \pm 0.3$ & $-3.0 \pm 0.1$ & $1.0 \pm 0.0$ & $13.1 \pm 0.1$ & $-2.2 \pm 0.4$ & $10.8 \pm 0.3$ \\
\hline
\end{tabular}


Table S25. Same as Table S1 but for compound 24.

\begin{tabular}{cccccccc}
\hline Residue & $\Delta \Delta \mathrm{E}_{\mathrm{vdw}}$ & $\Delta \Delta \mathrm{E}_{\text {ele }}$ & $\Delta \Delta \mathrm{GB}$ & $\Delta \Delta \mathrm{NP}$ & $\Delta \Delta \mathrm{H}$ & $\mathrm{IE}$ & $\Delta \Delta G_{\text {bind }}^{x \rightarrow a}$ \\
\hline Y105'A & $3.2 \pm 0.1$ & $-0.2 \pm 0.0$ & $-0.2 \pm 0.0$ & $0.3 \pm 0.0$ & $3.2 \pm 0.1$ & $-0.4 \pm 0.0$ & $2.8 \pm 0.0$ \\
$\mathrm{~N} 410 \mathrm{~A}$ & $1.7 \pm 0.1$ & $1.5 \pm 0.3$ & $-1.1 \pm 0.1$ & $0.1 \pm 0.0$ & $2.2 \pm 0.1$ & $-0.2 \pm 0.0$ & $2.0 \pm 0.1$ \\
$\mathrm{R} 425 \mathrm{~A}$ & $1.7 \pm 0.0$ & $-0.6 \pm 0.0$ & $0.6 \pm 0.0$ & $0.1 \pm 0.0$ & $1.7 \pm 0.0$ & $-0.0 \pm 0.0$ & $1.7 \pm 0.0$ \\
$\mathrm{~K} 409 \mathrm{~A}$ & $2.0 \pm 0.0$ & $1.1 \pm 0.1$ & $-1.0 \pm 0.0$ & $0.1 \pm 0.0$ & $1.4 \pm 0.1$ & $-0.2 \pm 0.0$ & $1.1 \pm 0.1$ \\
$\mathrm{~L} 166 \mathrm{~A}$ & $2.2 \pm 0.3$ & $0.0 \pm 0.0$ & $-0.4 \pm 0.0$ & $0.2 \pm 0.0$ & $2.0 \pm 0.3$ & $-1.0 \pm 0.1$ & $1.0 \pm 0.4$ \\
L172A & $0.7 \pm 0.0$ & $0.1 \pm 0.0$ & $-0.1 \pm 0.0$ & $0.0 \pm 0.0$ & $0.7 \pm 0.0$ & $-0.0 \pm 0.0$ & $0.7 \pm 0.0$ \\
Y106'A & $0.8 \pm 0.1$ & $0.0 \pm 0.0$ & $-0.0 \pm 0.0$ & $0.1 \pm 0.0$ & $0.9 \pm 0.1$ & $-0.4 \pm 0.1$ & $0.5 \pm 0.1$ \\
$\mathrm{H} 171 \mathrm{~A}$ & $0.5 \pm 0.0$ & $-0.1 \pm 0.0$ & $0.1 \pm 0.0$ & $0.0 \pm 0.0$ & $0.5 \pm 0.1$ & $-0.0 \pm 0.0$ & $0.5 \pm 0.0$ \\
E98'A & $0.5 \pm 0.0$ & $0.1 \pm 0.0$ & $-0.3 \pm 0.0$ & $0.1 \pm 0.0$ & $0.4 \pm 0.0$ & $-0.0 \pm 0.0$ & $0.4 \pm 0.0$ \\
\hline Total & $12.4 \pm 0.3$ & $1.9 \pm 0.2$ & $-2.4 \pm 0.0$ & $1.0 \pm 0.0$ & $12.9 \pm 0.2$ & $-2.3 \pm 0.2$ & $10.6 \pm 0.3$ \\
\hline
\end{tabular}


Table S26. Same as Table S1 but for compound 25.

\begin{tabular}{cccccccc}
\hline Residue & $\Delta \Delta \mathrm{E}_{\mathrm{vdw}}$ & $\Delta \Delta \mathrm{E}_{\text {ele }}$ & $\Delta \Delta \mathrm{GB}$ & $\Delta \Delta \mathrm{NP}$ & $\Delta \Delta \mathrm{H}$ & $\mathrm{IE}$ & $\Delta \Delta G_{\text {bind }}^{x \rightarrow a}$ \\
\hline Y105'A & $3.1 \pm 0.0$ & $-0.2 \pm 0.1$ & $-0.2 \pm 0.0$ & $0.3 \pm 0.0$ & $3.1 \pm 0.0$ & $-0.3 \pm 0.0$ & $2.7 \pm 0.1$ \\
$\mathrm{~N} 410 \mathrm{~A}$ & $1.7 \pm 0.1$ & $1.5 \pm 0.2$ & $-1.1 \pm 0.0$ & $0.1 \pm 0.0$ & $2.1 \pm 0.1$ & $-0.2 \pm 0.0$ & $2.0 \pm 0.1$ \\
$\mathrm{R} 425 \mathrm{~A}$ & $1.7 \pm 0.1$ & $-0.4 \pm 0.0$ & $0.4 \pm 0.0$ & $0.1 \pm 0.0$ & $1.8 \pm 0.1$ & $0.0 \pm 0.0$ & $1.8 \pm 0.1$ \\
$\mathrm{~K} 409 \mathrm{~A}$ & $1.2 \pm 0.0$ & $1.3 \pm 0.0$ & $-1.2 \pm 0.0$ & $0.1 \pm 0.0$ & $1.4 \pm 0.0$ & $-0.2 \pm 0.0$ & $1.2 \pm 0.1$ \\
L166A & $2.5 \pm 0.3$ & $0.0 \pm 0.0$ & $-0.6 \pm 0.1$ & $0.2 \pm 0.0$ & $2.1 \pm 0.3$ & $-0.9 \pm 0.2$ & $1.2 \pm 0.4$ \\
L172A & $0.6 \pm 0.0$ & $0.1 \pm 0.0$ & $-0.1 \pm 0.0$ & $0.0 \pm 0.0$ & $0.7 \pm 0.0$ & $0.0 \pm 0.0$ & $0.6 \pm 0.0$ \\
H171A & $0.5 \pm 0.0$ & $0.1 \pm 0.0$ & $0.0 \pm 0.0$ & $0.0 \pm 0.0$ & $0.5 \pm 0.0$ & $0.0 \pm 0.0$ & $0.5 \pm 0.0$ \\
Y106'A & $0.8 \pm 0.0$ & $0.1 \pm 0.0$ & $0.0 \pm 0.0$ & $0.1 \pm 0.0$ & $0.9 \pm 0.0$ & $-0.4 \pm 0.0$ & $0.5 \pm 0.0$ \\
E98'A & $0.5 \pm 0.0$ & $-0.1 \pm 0.0$ & $-0.1 \pm 0.0$ & $0.1 \pm 0.0$ & $0.3 \pm 0.0$ & $0.0 \pm 0.0$ & $0.3 \pm 0.0$ \\
I74A & $0.1 \pm 0.0$ & $0.0 \pm 0.0$ & $0.1 \pm 0.0$ & $0.0 \pm 0.0$ & $0.2 \pm 0.0$ & $0.0 \pm 0.0$ & $0.2 \pm 0.0$ \\
D168A & $0.0 \pm 0.0$ & $0.8 \pm 0.0$ & $-0.7 \pm 0.0$ & $0.0 \pm 0.0$ & $0.2 \pm 0.0$ & $0.0 \pm 0.0$ & $0.2 \pm 0.0$ \\
\hline Total & $12.7 \pm 0.3$ & $3.1 \pm 0.2$ & $-3.5 \pm 0.1$ & $1.0 \pm 0.0$ & $13.2 \pm 0.3$ & $-2.1 \pm 0.2$ & $11.2 \pm 0.5$ \\
\hline
\end{tabular}


Table S27. Same as Table S1 but for compound 26.

\begin{tabular}{|c|c|c|c|c|c|c|c|}
\hline Residue & $\Delta \Delta \mathrm{E}_{\mathrm{vdw}}$ & $\Delta \Delta \mathrm{E}_{\text {ele }}$ & $\Delta \Delta \mathrm{GB}$ & $\Delta \Delta \mathrm{NP}$ & $\Delta \Delta \mathrm{H}$ & IE & $\Delta \Delta G_{\text {bind }}^{x \rightarrow a}$ \\
\hline Y105'A & $3.9 \pm 0.2$ & $-0.4 \pm 0.1$ & $-0.4 \pm 0.0$ & $0.4 \pm 0.0$ & $3.4 \pm 0.2$ & $-0.4 \pm 0.1$ & $3.0 \pm 0.3$ \\
\hline $\mathrm{R} 425 \mathrm{~A}$ & $1.8 \pm 0.0$ & $0.1 \pm 0.1$ & $-0.1 \pm 0.1$ & $0.1 \pm 0.0$ & $1.9 \pm 0.0$ & $0.0 \pm 0.0$ & $1.8 \pm 0.0$ \\
\hline H171A & $1.3 \pm 0.1$ & $0.4 \pm 0.0$ & $-0.4 \pm 0.0$ & $0.0 \pm 0.0$ & $1.3 \pm 0.1$ & $0.0 \pm 0.0$ & $1.4 \pm 0.1$ \\
\hline N410A & $1.0 \pm 0.1$ & $1.1 \pm 0.7$ & $-0.7 \pm 0.3$ & $0.1 \pm 0.0$ & $1.5 \pm 0.2$ & $-0.4 \pm 0.2$ & $1.1 \pm 0.1$ \\
\hline E98'A & $1.4 \pm 0.1$ & $-0.2 \pm 0.0$ & $-0.3 \pm 0.1$ & $0.2 \pm 0.0$ & $1.1 \pm 0.1$ & $0.0 \pm 0.0$ & $1.1 \pm 0.1$ \\
\hline L172A & $0.9 \pm 0.0$ & $0.1 \pm 0.0$ & $-0.1 \pm 0.0$ & $0.1 \pm 0.0$ & $1.0 \pm 0.0$ & $-0.1 \pm 0.0$ & $0.9 \pm 0.1$ \\
\hline K409A & $0.8 \pm 0.0$ & $1.0 \pm 0.1$ & $-1.0 \pm 0.1$ & $0.1 \pm 0.0$ & $0.9 \pm 0.0$ & $0.0 \pm 0.0$ & $0.8 \pm 0.0$ \\
\hline N408A & $1.0 \pm 0.0$ & $0.3 \pm 0.0$ & $-0.4 \pm 0.0$ & $0.1 \pm 0.0$ & $0.9 \pm 0.0$ & $-0.1 \pm 0.0$ & $0.8 \pm 0.0$ \\
\hline S226A & $0.5 \pm 0.0$ & $0.4 \pm 0.1$ & $-0.1 \pm 0.0$ & $0.0 \pm 0.0$ & $0.7 \pm 0.1$ & $-0.1 \pm 0.0$ & $0.6 \pm 0.1$ \\
\hline L166A & $1.8 \pm 0.2$ & $0.0 \pm 0.0$ & $-0.3 \pm 0.1$ & $0.2 \pm 0.0$ & $1.7 \pm 0.2$ & $-1.1 \pm 0.4$ & $0.6 \pm 0.6$ \\
\hline F320'A & $0.5 \pm 0.0$ & $0.0 \pm 0.0$ & $-0.2 \pm 0.0$ & $0.0 \pm 0.0$ & $0.3 \pm 0.0$ & $0.0 \pm 0.0$ & $0.3 \pm 0.0$ \\
\hline $\mathrm{I} 74 \mathrm{~A}$ & $0.1 \pm 0.0$ & $0.0 \pm 0.0$ & $0.1 \pm 0.0$ & $0.0 \pm 0.0$ & $0.2 \pm 0.0$ & $0.0 \pm 0.0$ & $0.2 \pm 0.0$ \\
\hline $\mathrm{H} 254 \mathrm{~A}$ & $0.2 \pm 0.0$ & $0.0 \pm 0.0$ & $0.0 \pm 0.0$ & $0.0 \pm 0.0$ & $0.2 \pm 0.0$ & $0.0 \pm 0.0$ & $0.2 \pm 0.0$ \\
\hline Total & $15.0 \pm 1.1$ & $2.8 \pm 0.6$ & $-3.9 \pm 0.3$ & $1.2 \pm 0.1$ & $15.1 \pm 1.0$ & $-2.3 \pm 0.6$ & $12.8 \pm 0.6$ \\
\hline
\end{tabular}


Table S28. Same as Table S1 but for compound 27.

\begin{tabular}{|c|c|c|c|c|c|c|c|}
\hline Residue & $\Delta \Delta \mathrm{E}_{\mathrm{vdw}}$ & $\Delta \Delta \mathrm{E}_{\text {ele }}$ & $\Delta \Delta \mathrm{GB}$ & $\Delta \Delta \mathrm{NP}$ & $\Delta \Delta \mathrm{H}$ & IE & $\Delta \Delta G_{\text {bind }}^{x \rightarrow a}$ \\
\hline Y105'A & $3.3 \pm 0.1$ & $-0.2 \pm 0.1$ & $-0.3 \pm 0.0$ & $0.3 \pm 0.0$ & $3.2 \pm 0.1$ & $-0.3 \pm 0.0$ & $2.8 \pm 0.1$ \\
\hline $\mathrm{N} 410 \mathrm{~A}$ & $1.8 \pm 0.0$ & $1.3 \pm 0.1$ & $-1.1 \pm 0.0$ & $0.1 \pm 0.0$ & $2.2 \pm 0.0$ & $-0.2 \pm 0.0$ & $2.0 \pm 0.0$ \\
\hline $\mathrm{R} 425 \mathrm{~A}$ & $1.8 \pm 0.0$ & $-0.5 \pm 0.0$ & $0.5 \pm 0.0$ & $0.1 \pm 0.0$ & $1.9 \pm 0.0$ & $-0.0 \pm 0.0$ & $1.8 \pm 0.0$ \\
\hline K409A & $1.1 \pm 0.1$ & $2.2 \pm 0.1$ & $-2.1 \pm 0.1$ & $0.3 \pm 0.0$ & $1.4 \pm 0.1$ & $-0.2 \pm 0.0$ & $1.3 \pm 0.1$ \\
\hline L166A & $2.0 \pm 0.1$ & $-0.1 \pm 0.0$ & $-0.3 \pm 0.0$ & $0.2 \pm 0.0$ & $1.8 \pm 0.1$ & $-1.1 \pm 0.1$ & $0.8 \pm 0.2$ \\
\hline L172A & $0.6 \pm 0.0$ & $0.0 \pm 0.0$ & $-0.0 \pm 0.0$ & $0.0 \pm 0.0$ & $0.6 \pm 0.0$ & $-0.0 \pm 0.0$ & $0.6 \pm 0.0$ \\
\hline H171A & $0.5 \pm 0.0$ & $-0.2 \pm 0.0$ & $0.2 \pm 0.0$ & $0.0 \pm 0.0$ & $0.6 \pm 0.0$ & $-0.0 \pm 0.0$ & $0.6 \pm 0.0$ \\
\hline E98'A & $0.5 \pm 0.0$ & $0.0 \pm 0.0$ & $-0.2 \pm 0.0$ & $0.1 \pm 0.0$ & $0.4 \pm 0.0$ & $-0.0 \pm 0.0$ & $0.4 \pm 0.0$ \\
\hline Y106'A & $0.7 \pm 0.1$ & $0.1 \pm 0.0$ & $-0.0 \pm 0.0$ & $0.1 \pm 0.0$ & $0.9 \pm 0.1$ & $-0.5 \pm 0.0$ & $0.4 \pm 0.1$ \\
\hline $\mathrm{I} 74 \mathrm{~A}$ & $0.1 \pm 0.0$ & $0.0 \pm 0.0$ & $0.1 \pm 0.0$ & $0.0 \pm 0.0$ & $0.2 \pm 0.0$ & $0.0 \pm 0.0$ & $0.2 \pm 0.0$ \\
\hline Total & $12.5 \pm 0.2$ & $2.7 \pm 0.1$ & $-3.3 \pm 0.0$ & $1.1 \pm 0.0$ & $13.1 \pm 0.3$ & $-2.3 \pm 0.1$ & $10.8 \pm 0.2$ \\
\hline
\end{tabular}


Table S29. Same as Table S1 but for compound 28.

\begin{tabular}{cccccccc}
\hline Residue & $\Delta \Delta \mathrm{E}_{\mathrm{vdw}}$ & $\Delta \Delta \mathrm{E}_{\text {ele }}$ & $\Delta \Delta \mathrm{GB}$ & $\Delta \Delta \mathrm{NP}$ & $\Delta \Delta \mathrm{H}$ & $\mathrm{IE}$ & $\Delta \Delta G_{\text {bind }}^{x \rightarrow a}$ \\
\hline Y105'A & $3.5 \pm 0.1$ & $-0.1 \pm 0.0$ & $-0.4 \pm 0.0$ & $0.4 \pm 0.0$ & $3.4 \pm 0.2$ & $-0.3 \pm 0.1$ & $3.1 \pm 0.1$ \\
$\mathrm{~N} 410 \mathrm{~A}$ & $1.8 \pm 0.1$ & $1.2 \pm 0.1$ & $-0.9 \pm 0.0$ & $0.1 \pm 0.0$ & $2.1 \pm 0.0$ & $-0.2 \pm 0.0$ & $1.9 \pm 0.0$ \\
$\mathrm{R} 425 \mathrm{~A}$ & $1.8 \pm 0.1$ & $-0.2 \pm 0.0$ & $0.2 \pm 0.0$ & $0.1 \pm 0.0$ & $1.8 \pm 0.0$ & $0.0 \pm 0.0$ & $1.8 \pm 0.0$ \\
$\mathrm{~L} 166 \mathrm{~A}$ & $2.2 \pm 0.1$ & $0.0 \pm 0.0$ & $-0.3 \pm 0.0$ & $0.2 \pm 0.0$ & $2.0 \pm 0.1$ & $-0.9 \pm 0.1$ & $1.1 \pm 0.1$ \\
$\mathrm{~K} 409 \mathrm{~A}$ & $1.1 \pm 0.1$ & $1.1 \pm 0.0$ & $-1.1 \pm 0.0$ & $0.2 \pm 0.0$ & $1.3 \pm 0.1$ & $-0.2 \pm 0.1$ & $1.1 \pm 0.2$ \\
L172A & $0.6 \pm 0.0$ & $0.0 \pm 0.0$ & $-0.1 \pm 0.0$ & $0.0 \pm 0.0$ & $0.6 \pm 0.0$ & $0.0 \pm 0.0$ & $0.6 \pm 0.1$ \\
H171A & $0.5 \pm 0.0$ & $0.2 \pm 0.0$ & $-0.1 \pm 0.0$ & $0.0 \pm 0.0$ & $0.6 \pm 0.0$ & $0.0 \pm 0.0$ & $0.6 \pm 0.0$ \\
Y106'A & $0.7 \pm 0.1$ & $0.0 \pm 0.0$ & $0.0 \pm 0.0$ & $0.1 \pm 0.0$ & $0.8 \pm 0.1$ & $-0.4 \pm 0.0$ & $0.4 \pm 0.1$ \\
E98'A & $0.5 \pm 0.0$ & $-0.2 \pm 0.0$ & $0.0 \pm 0.0$ & $0.1 \pm 0.0$ & $0.4 \pm 0.0$ & $0.0 \pm 0.0$ & $0.3 \pm 0.0$ \\
I74A & $0.1 \pm 0.0$ & $0.0 \pm 0.0$ & $0.1 \pm 0.0$ & $0.0 \pm 0.0$ & $0.2 \pm 0.0$ & $0.0 \pm 0.0$ & $0.2 \pm 0.0$ \\
D168A & $0.0 \pm 0.0$ & $0.6 \pm 0.0$ & $-0.4 \pm 0.0$ & $0.0 \pm 0.0$ & $0.2 \pm 0.0$ & $0.0 \pm 0.0$ & $0.2 \pm 0.0$ \\
\hline Total & $12.9 \pm 0.3$ & $2.5 \pm 0.3$ & $-3.1 \pm 0.3$ & $1.1 \pm 0.1$ & $13.4 \pm 0.3$ & $-2.2 \pm 0.1$ & $11.2 \pm 0.3$ \\
\hline
\end{tabular}


Table S30. The average RMSDs of 28 ligands as well as their standard deviations in production step of molecular dynamics.

\begin{tabular}{|c|c|c|c|c|c|c|c|}
\hline $\begin{array}{c}\text { Compound } \\
\text { ID }\end{array}$ & RMSD & $\begin{array}{c}\text { Compound } \\
\text { ID }\end{array}$ & RMSD & $\begin{array}{c}\text { Compound } \\
\text { ID }\end{array}$ & RMSD & $\begin{array}{c}\text { Compound } \\
\text { ID }\end{array}$ & RMSD \\
\hline \multirow{3}{*}{1} & $1.2 \pm 0.2$ & \multirow{3}{*}{8} & $1.0 \pm 0.3$ & \multirow{3}{*}{15} & $0.9 \pm 0.3$ & \multirow{3}{*}{22} & $1.3 \pm 0.2$ \\
\hline & $1.0 \pm 0.3$ & & $0.9 \pm 0.2$ & & $1.0 \pm 0.2$ & & $1.0 \pm 0.3$ \\
\hline & $1.1 \pm 0.2$ & & $0.9 \pm 0.3$ & & $1.0 \pm 0.2$ & & $1.1 \pm 0.3$ \\
\hline \multirow{3}{*}{2} & $1.0 \pm 0.3$ & \multirow{3}{*}{9} & $1.3 \pm 0.3$ & \multirow{3}{*}{16} & $1.2 \pm 0.3$ & \multirow{3}{*}{23} & $0.5 \pm 0.1$ \\
\hline & $1.1 \pm 0.3$ & & $1.6 \pm 0.4$ & & $\begin{array}{l}0.9 \pm \\
0.3\end{array}$ & & $0.5 \pm 0.2$ \\
\hline & $0.9 \pm 0.2$ & & $1.5 \pm 0.3$ & & $0.9 \pm 0.3$ & & $0.4 \pm 0.2$ \\
\hline \multirow{3}{*}{3} & $1.1 \pm 0.2$ & \multirow{3}{*}{10} & $0.5 \pm 0.2$ & \multirow{3}{*}{17} & $0.9 \pm 0.2$ & \multirow{3}{*}{24} & $1.0 \pm 0.4$ \\
\hline & $0.9 \pm 0.2$ & & $0.5 \pm 0.2$ & & $0.8 \pm 0.3$ & & $1.1 \pm 0.4$ \\
\hline & $1.0 \pm 0.3$ & & $0.5 \pm 0.2$ & & $1.0 \pm 0.2$ & & $1.5 \pm 0.4$ \\
\hline \multirow{3}{*}{4} & $2.3 \pm 0.5$ & \multirow{3}{*}{11} & $1.2 \pm 0.2$ & \multirow{3}{*}{18} & $0.8 \pm 0.4$ & \multirow{3}{*}{25} & $0.5 \pm 0.2$ \\
\hline & $2.4 \pm 1.0$ & & $0.5 \pm 0.2$ & & $0.7 \pm 0.4$ & & $0.8 \pm 0.2$ \\
\hline & $1.5 \pm 0.3$ & & $0.6 \pm 0.3$ & & $0.6 \pm 0.3$ & & $0.6 \pm 0.2$ \\
\hline \multirow{3}{*}{5} & $1.1 \pm 0.3$ & \multirow{3}{*}{12} & $1.0 \pm 0.3$ & \multirow{3}{*}{19} & $1.1 \pm 0.2$ & \multirow{3}{*}{26} & $0.5 \pm 0.1$ \\
\hline & $1.2 \pm 0.2$ & & $2.1 \pm 0.2$ & & $0.9 \pm 0.2$ & & $1.0 \pm 0.3$ \\
\hline & $1.0 \pm 0.2$ & & $1.6 \pm 0.3$ & & $1.0 \pm 0.2$ & & $0.4 \pm 0.1$ \\
\hline \multirow{3}{*}{6} & $0.9 \pm 0.3$ & \multirow{3}{*}{13} & $1.4 \pm 0.2$ & \multirow{3}{*}{20} & $1.3 \pm 0.2$ & \multirow{3}{*}{27} & $0.9 \pm 0.2$ \\
\hline & $0.8 \pm 0.2$ & & $1.0 \pm 0.3$ & & $1.2 \pm 0.2$ & & $0.8 \pm 0.2$ \\
\hline & $0.9 \pm 0.2$ & & $1.3 \pm 0.2$ & & $1.3 \pm 0.2$ & & $0.8 \pm 0.2$ \\
\hline \multirow{3}{*}{7} & $2.6 \pm 0.5$ & \multirow{3}{*}{14} & $1.2 \pm 0.2$ & \multirow{3}{*}{21} & $0.9 \pm 0.4$ & \multirow{3}{*}{28} & $0.5 \pm 0.1$ \\
\hline & $2.1 \pm 0.4$ & & $1.0 \pm 0.3$ & & $1.0 \pm 0.3$ & & $0.7 \pm 0.2$ \\
\hline & $2.5 \pm 0.5$ & & $1.3 \pm 0.2$ & & $0.7 \pm 0.4$ & & $0.7 \pm 0.1$ \\
\hline
\end{tabular}

